\title{
Der Konjunktiv II in Salzburger Varietäten: \\ Grammatik, Gebrauch, soziale Faktoren
}

Konstantin Niehaus, Irmtraud Kaiser und Peter Mauser (Salzburg)

\begin{abstract}
If one looks for linguistic information on the past subjunctive (Konjunktiv II) in Salzburg, there is not much to obtain. A few studies have commented on its morphology in dialect (Mauser 1998 und 2021a), the atlases of colloquial German (Eichhoff 1977-2000; Elspaß/Möller 2003-) provide some general insights as to the use of analytical forms, other studies aim at a broader scope on Bavarian dialects (e. g. Wiesinger 1989; Donhauser 1992). This article gives a first more detailed account. We present findings from a traditional dialectological investigation (relying on on-site interviews using a questionnaire) as well as a variationist analysis of online questionnaire data on colloquial Salzburg varieties. The dialectological analysis focuses on language-internal factors such as analytic vs. synthetic construction and variation of endings but also shows strong differences between the northern Flachgau, which surrounds the capital of Salzburg, and the remote and more rural southern Lungau. The online questionnaire allows for a quantitative inspection of the use of Konjunktiv II forms of brauchen, i. e. analytical vs. synthetical, (non-)use of umlaut, inflection with -ert/-at and -te. Statistical analysis shows that the participants' preferences correlate with their age, region of origin, degree of education, and mobility: Younger speakers indicate the use of analytical forms more frequently, and standard (or near-standard) forms are favoured by participants near or directly from the city of Salzburg and those of higher education and mobility. In the end, we will shortly discuss the comparability of our outcomes and future research avenues, especially with regard to the Salzburg region and its neighbouring dialect areas.
\end{abstract}

\section{$1 \quad$ Konjunktiv II in Salzburg: Motivation und Zielsetzung dieses Beitrags}

Das Bundesland Salzburg zeigt wie die meisten österreichischen Bundesländer immer noch einen stabilen Gebrauch der Basisdialekte, die in diesem Fall sowohl zu den westmittel-, als auch südmittel- und südbairischen gezählt werden können (cf. Lenz 2019). In einem Kontinuum des Varietätengebrauchs (Diaglossie) werden aber auch Standard- und standardnahe sowie regiolektale (oder großräumig ,umgangssprachliche“) Formen verwendet; diese vertikale Varietätenstruktur in Österreich ist jedoch bislang vergleichsweise schlecht erforscht (cf. ibd.: 319). ${ }^{1}$

\footnotetext{
${ }^{1}$ Auf die Diskussion, ob es sich tatsächlich um ein Kontinuum oder vielmehr um verschiedene Sprechlagen (oder Varietäten) handelt, kann hier nicht näher eingegangen werden (cf. u. a. Scheutz 1999). Zum aktuellen Zeitpunkt
} 
Gleiches gilt auf horizontaler dialektaler Ebene für Variation und Wandel der Morphosyntax (cf. ibd.: 334). Für das Land Salzburg ist dieser Forschungsmangel ebenso offensichtlich wie unverständlich, ist doch angesichts seiner dialektalen Übergangsgebiete und seiner zentralen Lage innerhalb Österreichs eine hohe soziolinguistische Dynamik zu erwarten.

Wir möchten auf regionaler Ebene mit neuer Evidenz dazu beitragen, diese Lücken hinsichtlich der horizontalen wie vertikalen Variation zu schließen. Der Beitrag präsentiert deswegen sowohl Ergebnisse variationslinguistischer als auch dialektologischer Analysen, die dezidiert ein bisher unterrepräsentiertes morphosyntaktisches Phänomen behandeln, nämlich den Gebrauch des Konjunktivs II im Land Salzburg. Der Beitrag versteht sich dabei als erste empirische Umschau auf die aktuelle Situation der Variationsebenen in diesem Gebiet und konzentriert sich auf soziale wie grammatische Faktoren, wobei die Morphologie die Basis der Betrachtung bildet. Die Darstellung vereint zwei methodisch unterschiedlich erhobene Datensätze aus aktuell laufenden Forschungsprojekten für einerseits die vertikale, andererseits die horizontale Variationsebene, die nichtsdestoweniger wertvolle Einblicke in die jüngere und aktuelle Verwendung des Konjunktivs II in Salzburg geben können.

Auf Basis der bislang verfügbaren Erkenntnisse zu theoretisch möglichen morphologischen Konstruktionsvarianten des Konjunktivs II (phonetisch-phonologische Aspekte können hier nur gestreift werden) werden wir zunächst eine Fallstudie zu Sprachaufnahmen aus Ortserhebungen im Flachgau (Bezirk Salzburg-Umgebung) und Lungau (Bezirk Tamsweg) vorstellen. Sie fokussiert neben sprachinternen Faktoren - etwa dem Einfluss des verwendeten Verbs - auch regionale Unterschiede. Die geografische Verteilung der Aufnahmen ist hierbei günstig, weil zum einen der Flachgau infrastrukturell eine gute oder sogar sehr gute Anbindung an die Landeshauptstadt hat und viele Bewohner*innen entsprechend mobil sind (z. B. Pendler*innen, cf. Land Salzburg 2012), zum anderen der Lungau als Beispiel für eine entlegene rurale Region gelten kann. Insofern ist eine breite Darstellung auch auf dialektal-horizontaler Ebene bis zu einem gewissen Grad möglich. Eine zweite Fallstudie widmet sich hingegen der variationslinguistisch-quantitativen Analyse von Daten aus einer großen Online-Umfrage zum Konjunktiv II von brauchen, konkret der Überprüfung von Hypothesen insbesondere mit Hinblick auf Wohnregion, Mobilität, Alter und Bildung der Sprecher*innen. Zu jeder Studie werden wir die unterschiedlichen Datensätze und methodischen Zugänge separat beschreiben.

Schließlich werden die Ergebnisse, soweit möglich, verglichen (vor allem in Bezug auf die variationslinguistischen Hypothesen) und mögliche Anknüpfungspunkte für künftige Forschung ausgelotet.

\section{Theorie: Zur Morphologie der Konjunktiv-II-Varianten im bairischen Raum}

Aus Platzgründen können wir hier nicht auf die gesamte bestehende Forschung zur Morphologie des Konjunktivs in den bairischen Dialekten eingehen. Einige basale Ausführungen zur Variation müssen deshalb an dieser Stelle genügen.

kann über diese Frage mangels empirischer Grundlage wohl kaum entschieden werden und es ist zudem zu erwarten, dass sie regional, lokal und letztlich individuell unterschiedlich zu beantworten sein wird (cf. Ender/Kaiser 2014; Vergeiner 2019). 
Grundsätzlich interferieren bei der Bildung des Konjunktivs II die beiden Kodierungsverfahren der Analyse und Synthese. Für die synthetischen Kodierungsverfahren ergibt sich eine Unterscheidung auf Basis von Suffix- und/oder Binnenflexion (i. e. Stammmodifikation). Für die analytischen Kodierungsverfahren lässt sich eine Differenzierung auf Basis der verwendeten Auxiliare vornehmen. Für synthetische Verfahren differenziert Mauser (1998: 343) in eine „schwache“ (i. e. suffixflektierende) und „kontaminierte“ (i. e. stammmodifizierende und suffixflektierende) Bildungsweise - die sich aufgrund der Suffigierung aber auch als eine gemeinsame Kategorie sehen lassen - sowie eine „starke“, nur stammmodifizierende Bildungsweise. Orientiert hieran sowie weiters an Wiesinger (1989: 56) und Glauninger (2008: 233) lassen sich so prinzipiell folgende Formtypen (Varianten) des Konjunktivs II festhalten, die vertikal wie horizontal unterschiedlich verteilt (und unterschiedlich in der Lautung realisiert) ${ }^{2}$ sein können (Beispiele im Folgenden in 1.Sg.Konj.Prät. von nehmen):

(1) - $\varnothing$ stammmodifizierende Nullmorphemvariante (stark synthetisch), z. B. i na:m-Ø

(2) -at (schwach oder kontaminiert synthetisch, -ad/-at-Konjunktiv) mit Stammvokalmodifikation, z. B. i nem-at, oder ohne, z. B. i na:m-at

(3) tun + Inf. (analytisch, täte-Konjunktiv), z. B. i da:d(-at) ne:ma

(4) würde + Inf. (analytisch, würde-Konjunktiv), z. B. i wüad(-at)/wuad(-at) ne:ma

Formtyp 4 wird (mittlerweile) als morphologische Standardvariante betrachtet (cf. FabriciusHansen 2016: 542-543, 553), ebenso wie die synthetische Standardvariante auf Basis des Präteritalstamms ich nähme (cf. ibd.: 547), die grundsätzlich Formtyp 1 zuzurechnen ist. ${ }^{3}$

Eine umfassende vertikale und horizontale Behandlung des Konjunktivgebrauchs in Salzburg ist ausständig. Einige dialektologische Studien (i. e. über Ortserhebungen per Interview oder Fragebogen bzw. Fragebuch) beschäftigen sich mit der dialektalen Morphologie des Konjunktivs in Salzburg (z. B. Mauser 1998 und 2021a; Sommerauer 2009) und lassen eine gewisse regionale Variation erwarten, etwa vor dem Hintergrund der sprachgeschichtlichen Zusammenhänge für das konservativere Südbairische in Salzburg ein stärkeres Beharren auf Formtyp 1. ${ }^{4}$ In etwas großräumigerer Perspektive müssen aus dem dialektologischen Forschungszusammenhang weiters Zehetner (1983) und Wiesinger (1989) für das Bairische in übergreifender Betrachtung sowie Beiträge zum Mittelbairischen in Österreich wie Mindl (1924/25) für Oberösterreich und Glauningers Arbeiten (z. B. Glauninger 2011a) für Wien genannt werden. Über noch breiter angelegte Erhebungen wie die Atlanten zur Umgangs- und Alltagssprache (Eichhoff 1977-2000, Elspaß/Möller 2003-) erfährt man zudem Generelles zur Verwendung

\footnotetext{
${ }^{2}$ Im Sprachgebrauch, i. e. an der Schnittstelle zur Pragmatik, können zudem die Funktionsunterschiede zwischen Irrealis, Potentialis und Höflichkeitsausdruck die Formbildung beeinflussen (cf. Fabricius-Hansen 2016: 533; auch Glauninger 2011b zum „,honorativen Konjunktiv II“).

${ }^{3} \mathrm{Zu}$ Fragen der Norm und Variation beim Konjunktiv II cf. etwa auch Lotze/Gallmann (2009).

${ }^{4}$ Eine Darstellung der Entstehung der verschiedenen Formtypen kann hier nicht vorgenommen werden; vergleiche zu den synthetischen Konjunktiv-II-Periphrasen überblicksartig Fleischer/Schallert (2011) bzw. Eroms (1998), Fischer (2001) und Smirnova (2006); zur Entstehung und Ausbreitung des Konjunktivsuffes -at/-ad cf. Reiffenstein (1968: 174-176) und Schirmunski (1962: 511-514). - Zur Zugrundelegung des Axioms der Konservativität des Südbairischen bedürfte es einer eigenständigen Studie; die Annahme der „Beharrsamkeit“ des Südens findet sich etwa bei Kranzmayer (1956: Einleitung et passim); für das Land Salzburg, stärker differenzierend, Reiffenstein (1955: 44-58).
} 
analytischer Formen in Regiolekten und standardnaher Sprache, wobei die Methodik bei Elspaß/Möller (2003-) einer jüngeren, quantitativ-soziolinguistischen Vorgehensweise per indirekter Befragung entspricht. ${ }^{5}$ Zuletzt sei auch eine aktuelle experimentelle Studie (Breuer/Wittibschlager 2020: 153-154) erwähnt, die relevante Ergebnisse zum Konjunktivgebrauch im gesamten bairischen Raum Österreichs vorweisen kann: Synthetische Formen mit dem Suffix -at/-ad (Formtyp 2) sind demnach vor allem bei irregulären Verben verbreitet (cf. hierzu auch Wiesinger 1989: 56-61), bei Betrachtung des gesamten vertikalen Variationsspektrums scheinen jedoch insgesamt analytische Konstruktionen mit würde oder täte (Formtypen 3 und 4) zu dominieren (cf. auch Glauninger 2011a: 100) - dies gilt erst recht, wenn Standardsprache intendiert wird (cf. Breuer/Wittibschlager 2020: 153-154). Analytische Konstruktionen werden zudem tendenziell von jüngeren Sprecher*innen verwendet, insbesondere solchen aus dem urbanen Raum (cf. ibd.: 159). Für Salzburg berücksichtigte die Studie allerdings nur den Belegort Hüttschlag (im Bezirk St. Johann, Pongau) mit neun jüngeren, mobilen (1835) und zwei älteren (60+), immobilen Sprecher*innen (cf. ibd.: 148). Allgemein ist jedoch aufgrund der wiederholt beobachteten Dialekt-zu-Standard-Advergenz in den (mittel-)bairischen Dialekten Österreichs (cf. referierend Lenz 2019: 338-349) von einer größeren Affinität zu standardnahen Konjunktivformen bei jüngeren und urbanen Sprecher*innen auszugehen. Ebenso gilt aller Voraussicht nach analog zu allgemeinen Variationstendenzen auch für den Konjunktiv II, dass sich höher gebildete und mobilere Personen eher standardnaher bzw. umgangssprachlicher als dialektaler Formen bedienen (cf. z. B. Steinegger 1998: 153-167 und 300-301).

\section{Dialektologische Ortserhebungen im Flachgau und Lungau}

\subsection{Datengrundlage}

Die traditionell dialektologisch mittels Fragebuch erhobenen Daten stammen aus zwei unterschiedlichen Erhebungsphasen (im Folgenden kurz EH I und EH II): In den Jahren 2002 bis 2006 wurden im Salzburger Flachgau 111 sowie im Lungau 48 Interviews durchgeführt. Die Erhebungsorte im Flachgau waren im agrarisch strukturierten Norden die Gemeinden St. Georgen, Dorfbeuern sowie die Industrieenklave Bürmoos, im Seengebiet der alte Tourismusort Mattsee und im Osten die sich durch die verkehrstechnisch günstige Lage in den letzten Jahrzehnten ungemein dynamisch entwickelnde Gemeinde Seekirchen und schließlich Straßwalchen, das aufgrund seiner Lage an der nordöstlichen Peripherie des Bezirks und starken Zergliederung in seiner agrarischen Prägung dem Flachgauer Norden nahesteht. Dialektgeografisch fallen alle erhobenen Gemeinden in den oben definierten Bereich des Westmittelbairischen. Während für den politischen Bezirk Salzburg-Umgebung (Flachgau) mit Blick auf Wirtschafts- und Sozialstruktur keine Einheitlichkeit angenommen werden darf, lässt sich im Lungau vergleichsweise hohe Homogenität feststellen, die sich aufgrund jahrhundertelanger Abgeschiedenheit dieses hochalpinen Beckens in starker (u. a. sprachlicher) Beharrsamkeit ${ }^{6}$ äußert. Neben Land- und Forstwirtschaft ist hier der (v. a. Winter-)Tourismus der bestimmende Wirtschaftsfaktor. Die Erhebungsorte im Lungau waren (abgesehen von einem Einzelinterview

\footnotetext{
5 Z. B. täte probieren, cf. Elspaß/Möller (2003-), Runde 2, Frage 18c.

${ }^{6}$ Cf. etwa in diesem Sinne Bauer (1967: § 5), Reiffenstein (1984) oder jüngst Mauser (2021a: 57-67).
} 
in Mauterndorf) Tamsweg (als Bezirkshauptstadt das Schul- und Verwaltungszentrum der Region), Mariapfarr, Lessach und Zederhaus. Der gesamte Lungau hebt sich dialektgeografisch von den im Norden des politischen Bezirkes angrenzenden südmittelbairischen Regionen ab und zeigt hohe Affinität zum Südbairischen. In Vermeidung des Terminus ,südsüdmittelbairisch“ ist in Mauser (2021a: 59) vom „eher Südbairischen“ die Rede. Unterschiede zeigen sich im gesamten politischen Bezirk kaum auf vertikaler, sondern vielmehr auf horizontaler Ebene. Sichtbar werden sie vor allem dadurch, dass im Unterschied zur Peripherie entlegener Seitentäler (etwas das Zederhaus- oder Lessachtal) im Zentralraum Prozesse der Regiolektalisierung stärker fortgeschritten sind (cf. bereits in diesem Sinn Bauer 1967: 31-35). Im Rahmen dieser EH I wurden Konjunktiv-II-Formen von 13 Verben - Vollverben (= Vv), falls nicht anders angegeben - erhoben: geben, haben, kommen, können (Modalverb), lassen, liegen, mögen (Modalverb), müssen (Modalverb), nähen, sehen, sein, tun, ziehen. In der EH II in den Jahren 2018 und 2019 wurden im Rahmen eines LEADER-Projekts neuerlich 55 Aufnahmen im Lungau (sowie 22 Aufnahmen in den an den Lungau angrenzenden Regionen Ennspongau, Obersteiermark und Mittelkärnten; allerdings unberücksichtigt in diesem Beitrag) gemacht, und zwar flächendeckend in allen Gemeinden des politischen Bezirks (cf. auch Mauser 2021a). Im Rahmen dieser Erhebung wurden über die in EH I erhobenen 13 Verben hinaus auch Konjunktiv-IIFormen folgender 6 Verben erfasst: dürfen (Modalverb), essen, haben (Auxiliar), helfen, lehnen, lesen. ${ }^{7}$ Die konkrete Verteilung der Alters- und Bildungsgruppen beider Erhebungen ist in Tabelle 1 zusammengefasst:

\begin{tabular}{|c|c|c|c|c|c|c|c|}
\hline & $\begin{array}{l}\text { Bür- } \\
\text { moos }\end{array}$ & $\begin{array}{l}\text { Dorf- } \\
\text { beuern }\end{array}$ & Mattsee & $\begin{array}{c}\text { St. } \\
\text { Georgen }\end{array}$ & $\begin{array}{c}\text { See- } \\
\text { kirchen }\end{array}$ & $\begin{array}{c}\text { Straß- } \\
\text { walchen }\end{array}$ & $\begin{array}{c}\text { Flachgau } \\
\text { gesamt } \\
\text { (EH I) }\end{array}$ \\
\hline \multicolumn{8}{|c|}{ Altersgruppen EH I (Flachgau) } \\
\hline 20-39 & 6 & 1 & 7 & 3 & 2 & 7 & 26 \\
\hline $40-59$ & 11 & 14 & 7 & 8 & 15 & 7 & 62 \\
\hline $60+$ & 10 & 3 & 3 & 1 & 4 & 2 & 23 \\
\hline \multicolumn{8}{|c|}{ Bildungsgruppe EH I (Flachgau) } \\
\hline Pflichtschule & 14 & 10 & 10 & 5 & 8 & 11 & 58 \\
\hline $\begin{array}{l}\text { Fach-/ } \\
\text { Handelsschule }\end{array}$ & 5 & 4 & 4 & 5 & 3 & 4 & 25 \\
\hline Matura & 5 & 1 & 2 & 2 & 5 & 1 & 16 \\
\hline Studium & 3 & 2 & 1 & 0 & 5 & 0 & 11 \\
\hline Doktorat & 0 & 1 & 0 & 0 & 0 & 0 & 1 \\
\hline Gesamt & 27 & 18 & 17 & 12 & 21 & 16 & 111 \\
\hline
\end{tabular}

\footnotetext{
${ }^{7}$ Die Echtzeitdiachronie (12 bis 18 Jahre) zwischen EH I und EH II blieb unberücksichtigt, außer bei der Analyse nach Altersgruppen. Bei der Analyse nach Altersgruppen wurde nur das Datenmaterial aus EH I ausgewertet, da es Datensätze aus beiden politischen Bezirken umfasst. Bei bezirksspezifischen Vergleichsanalysen zwischen Flachgau und Lungau wurde nur auf das Datenmaterial aus EH I zurückgegriffen.
} 


\begin{tabular}{|c|c|c|c|c|c|c|c|}
\hline & $\begin{array}{c}\text { Lessach } \\
\text { (EH I) }\end{array}$ & $\begin{array}{l}\text { Maria- } \\
\text { pfarr } \\
(\mathrm{EH} \mathrm{I})\end{array}$ & $\begin{array}{c}\text { Mautern- } \\
\text { dorf } \\
(\mathrm{EH} \mathrm{I})\end{array}$ & $\begin{array}{l}\text { Tams- } \\
\text { weg } \\
\text { (EH I) }\end{array}$ & $\begin{array}{l}\text { Zeder- } \\
\text { haus } \\
(\mathrm{EH} \mathrm{I})\end{array}$ & $\begin{array}{c}\text { Lungau } \\
\text { gesamt } \\
\text { (EH I) }\end{array}$ & $\begin{array}{c}\text { Lungau } \\
\text { gesamt } \\
\text { (EH II) }\end{array}$ \\
\hline \multicolumn{8}{|c|}{ Altersgruppen (Lungau) } \\
\hline $20-39$ & 7 & 3 & 0 & 6 & 3 & 19 & 13 \\
\hline $40-59$ & 3 & 9 & 0 & 8 & 8 & 28 & 13 \\
\hline $60+$ & 0 & 0 & 1 & 0 & 0 & 1 & 29 \\
\hline \multicolumn{8}{|c|}{ Bildungsgruppe (Lungau) } \\
\hline Pflichtschule & 6 & 5 & 0 & 6 & 4 & 21 & 32 \\
\hline $\begin{array}{l}\text { Fach-/ } \\
\text { Handelsschule }\end{array}$ & 1 & 2 & 1 & 3 & 4 & 11 & 14 \\
\hline Matura & 2 & 3 & 0 & 4 & 0 & 9 & 4 \\
\hline Studium & 0 & 2 & 0 & 1 & 3 & 6 & 5 \\
\hline Doktorat & 1 & 0 & 0 & 0 & 0 & 1 & $\mathbf{0}$ \\
\hline Gesamt & 10 & 12 & 1 & 14 & 11 & 48 & 55 \\
\hline
\end{tabular}

Tabelle 1: Übersicht Proband*innen EH I und II nach Alters- und Bildungsgruppen

Der Fokus in EH I lag auf der mittleren Generation (Durchschnittsalter Sprecher*innen: 47). Aufgrund der Zielsetzungen des Projekts (Dokumentation basisdialektaler Varietäten) war in EH II die Gruppe der Sprecher*innen 50+ deutlich stärker repräsentiert (Durchschnittsalter Sprecher*innen: 58). Die Verteilung der Geschlechter ist leicht asymmetrisch im Verhältnis 52,3\% : 47,6\% zugunsten männlicher Gewährspersonen (112 männliche, 102 weibliche Gewährspersonen).

In beiden Erhebungen wurden die Konjunktiv-II-Formen mittels Übersetzungs- (z. B. Wenn er mich doch endlich sähe!) bzw. vor allem Vervollständigungsaufgaben (z. B. Er ist die ganze Woche nicht gekommen! Wenn er doch ...) elizitiert. Bei den Erhebungen waren die entsprechenden Verben auf knapp 440 (EH I) bzw. 450 (EH II) Items verteilt, sodass bei durchschnittlicher Interviewdauer zwischen 90 und 120 Minuten mit geringem Paradigmendruck bei der Beantwortung gerechnet werden muss. Nach gängiger dialektologischer Praxis wurden suggerierte Formen und ggfs. Erinnerungsbelege besonders markiert. Alle entsprechenden Formen blieben hier unberücksichtigt. Während in der EH I parallel zur Aufnahme das gesamte Interview mittranskribiert wurde (und nachträglich aus dem Rohtranskript ein Reintranskript angefertigt wurde), unterblieb in EH II die Transkription. Zum Zweck leichterer Analyse und der Veröffentlichung im Rahmen einer sprechenden Landkarte (cf. Mauser 2020b) wurden die aufgezeichneten Interviews aus EH II allerdings mit der Signalverarbeitungssoftware STx (Sound Tool Extended) segmentiert. 
Folgende Verben wurden in EH I und/oder EH II im Konjunktiv II erhoben:

- $\quad$ starke/suppletive Verben: essen (K1. V), geben (Kl. V), helfen (Kl. IIIb), kommen (K1. IV), lassen (K1. red. Ib), lesen (K1. V), liegen (K1. V), sehen (K1. V), sein (Vv, suppl./K1. V), ${ }^{8}$ tun (Vv, suppl.), ${ }^{9}$ ziehen (Kl. IIb)

- schwache Verben: haben (Vv), haben (Aux.), lehnen, nähen

- Modalverben: dürfen, können, mögen, müssen

Für all diese morphologisch z. T. sehr unterschiedlichen Verben wurden in der Analyse die oben erwähnten 4 Formtypen berücksichtigt. Mit Blick auf die Analyse des dialektologischen Datenmaterials gilt es darüber hinaus festzuhalten, dass bei Formtyp 1 (stammmodifizierende Nullmorphemvariante) tatsächlich nicht nur um- und ablautbedingte vokalische Modifikationen Berücksichtigung fanden, sondern auch konsonantische Modifikationen (z. B. grammatischer Wechsel in ['scexn], sehen': ['sa:g(at)], Frikativierung vor Dental wie in mögen : möchte bis zu starker Suppletion wie in sein : wäre). Bei Formtyp 3 (tun + Inf.) wäre grundsätzlich eine Differenzierung beim Auxiliar zwischen täte (['dat]) und tätad (['da:dat 'da:rat] + Inf. möglich, die allerdings im Rahmen der vorliegenden Analyse keine weitere Berücksichtigung fand. Bei Formtyp 4 (würde + Inf.) wurden die basisdialektalen Realisationsvarianten ['vu:xt] + Infinitiv für den Lungau bzw. ['vuext] + Inf. für den Flachgau aufgrund ihrer außerordentlichen Salienz unterschieden von allen anderen Realisationsmöglichkeiten (das sind standardsprachliches ['vyed( $(\varepsilon)]$ bzw., ohne Umlaut, umgangssprachliches ['vuet]).

\subsection{Resultate: Verbgruppen und Analyse bzw. Synthese}

Zunächst zu den Modalverben. Diese zeigen in den dialektologischen Daten das folgende Bild:

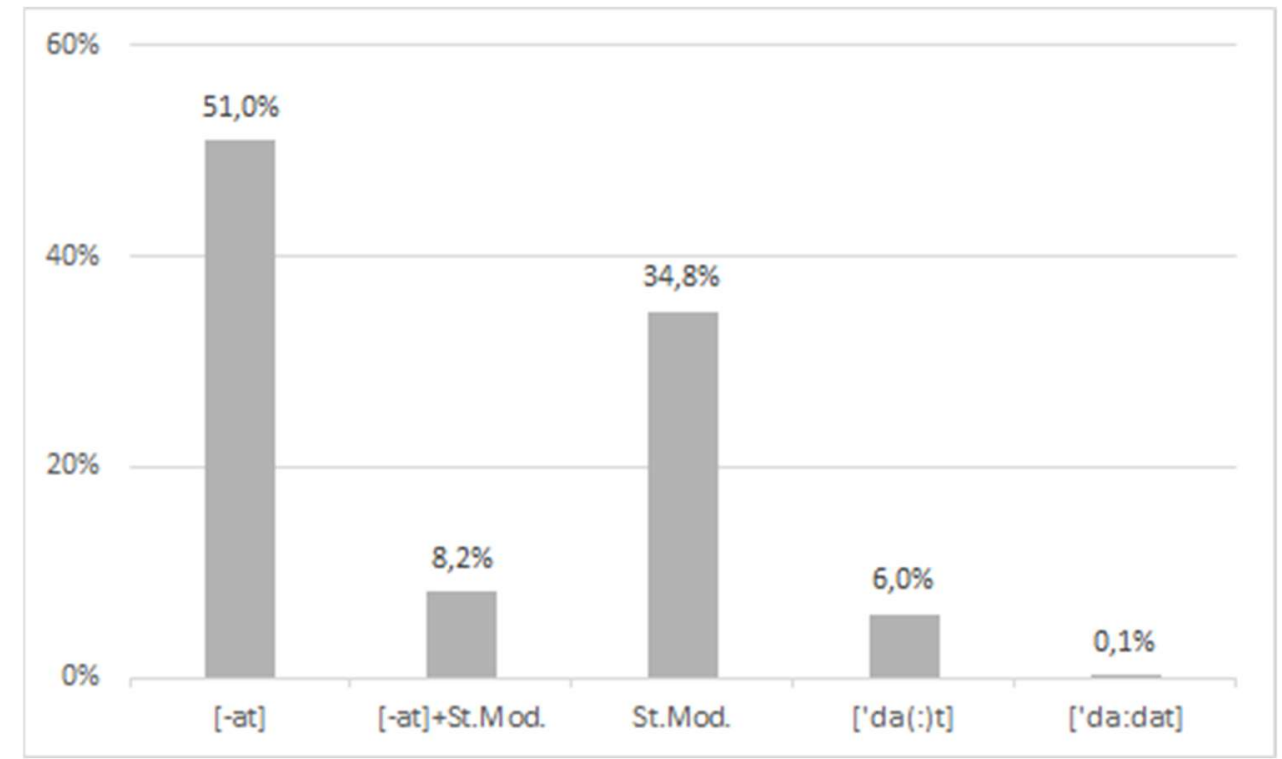

Abbildung 1: Konjunktiv-II-Bildung: Modalverben, Flach- und Lungau (EH I/II; n=736)

\footnotetext{
${ }^{8}$ Die in unserem Zusammenhang relevanten präteritalen Formen folgen historisch der Klasse V starker Bildung; cf. Braune (2004: § 378).

${ }^{9}$ Das Verb tun zeigt im Althochdeutschen morphologisch Merkmale sowohl reduplizierender als auch starker Verben K1. V; cf. Braune (2004: § 381).
} 
Die mit Abstand am häufigsten realisierte Form (375 Belege, 51,0\%) ist die Bildung nach Formtyp 2 mit dem Konjunktivmorphem -at (z. B. ['deefat] ,dürfte'), gefolgt von KonjunktivII-Formen nach Formtyp 1, i. e. mittels Stammmodifikation (= St.Mod.) (256 Belege, 34,8\%; z. B. ['mext] ,möchte'). In vergleichsweise wenigen Fällen kann das Morphem -at auch mit einer Modifikation des Stammes einhergehen (60 Belege, 8,2\%; z. B. ['khã:nat] ,könnte' zum Infinitiv [' $\left.{ }^{\mathrm{h}} \mathrm{i}: n a\right] ~\left[{ }^{\prime} \mathrm{k}\right.$ ẽe:na]). Selten ist die analytische Bildung nach Formtyp 3 anzutreffen (45 Belege, 6,1\%): ['dat] + Inf. (44 Belege) bzw. ['da:dat] + Inf. (1 Beleg). Formtyp 4 (würdePeriphrase) kommt gar nicht vor. Diese Zahlen können freilich nur eine grobe Orientierung geben - nicht für jedes der erhobenen Modalverben sind alle Formtypen realisiert bzw. v. a. realisierbar (z. B. sind teilweise keine Bildungen mit Stammmodifikation, etwa ['deefn] *['dueft] bzw. *['dueftat], anzutreffen). Die morphologisch höchste Vergleichbarkeit (schwache Präteritalbildung mit grundsätzlich modifizierbarem Stamm) mit dem in der nachfolgend präsentierten Online-Umfrage abgefragten Verb brauchen besteht mit dem Verb können:

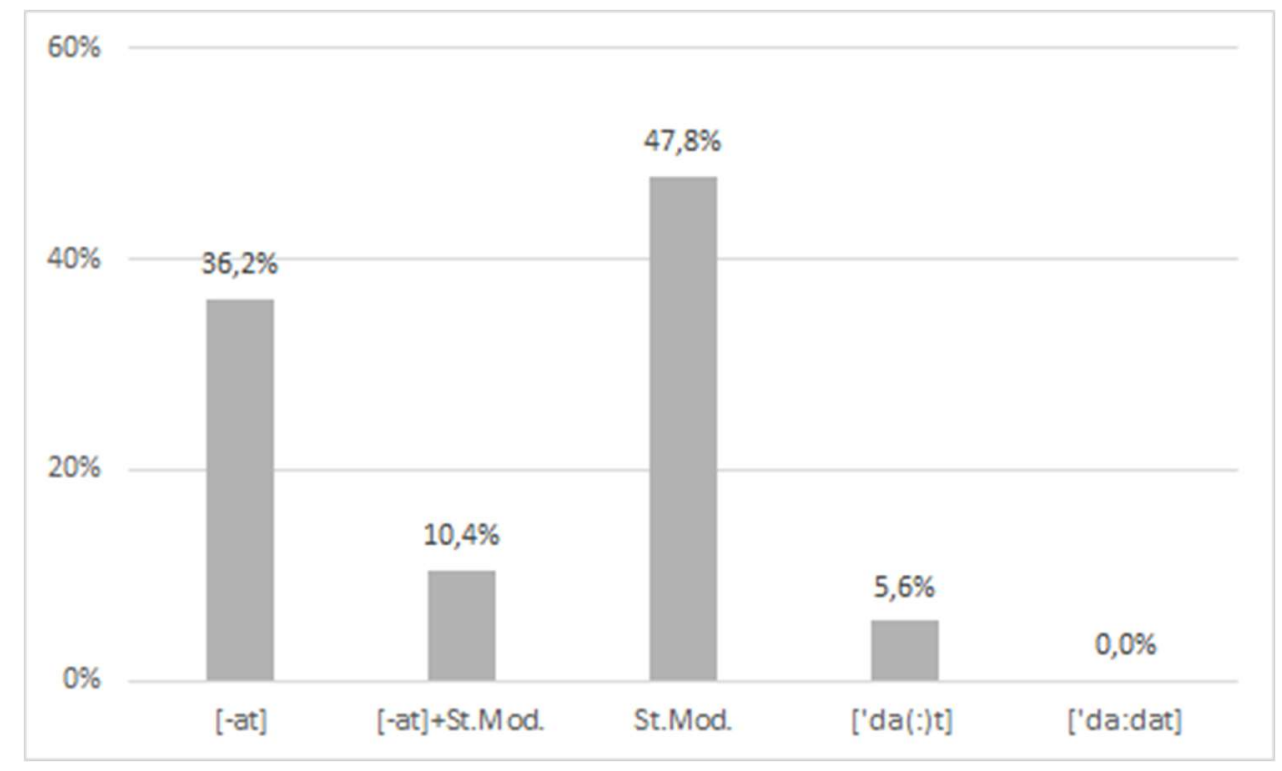

Abbildung 2: Konjunktiv-II-Bildung: können, Flach- und Lungau (EH I; n=268)

Das Verb können zeigt, im Vergleich zum Sample der 4 erhobenen Modalverben, einen geringeren Prozentsatz für die -at-Formen (z. B. ['khẽ:nat] ,könnte'; 97 Belege, 36,2\%) und zugleich einen entsprechend höheren Wert für die rein stammmodifizierende Bildung (z. B. [ ${ }^{\mathrm{k}} \mathrm{h}$ ãnt] ,könnte'; 128 Belege, 47,8\%). Das Verhältnis synthetischer (stammmodifizierender und/oder morphemflektierter) zu analytischer Konjunktiv-II-Bildung entspricht beim Verb können aber insgesamt dem Sample der 4 erhobenen Modalverben: 6,1\% (analytischer Formtyp; 45 Belege) zu 93,9\% (synthetischer Formtyp; 691 Belege) beim gesamten Modalverbensample und 5,6\% (15 Belege) zu 94,4\% (253 Belege) beim Verb können.

Nimmt man das gesamte Sample aller Verben in den Fokus, so zeigen sich für Flach- und Lungau die folgenden Verteilungen: 


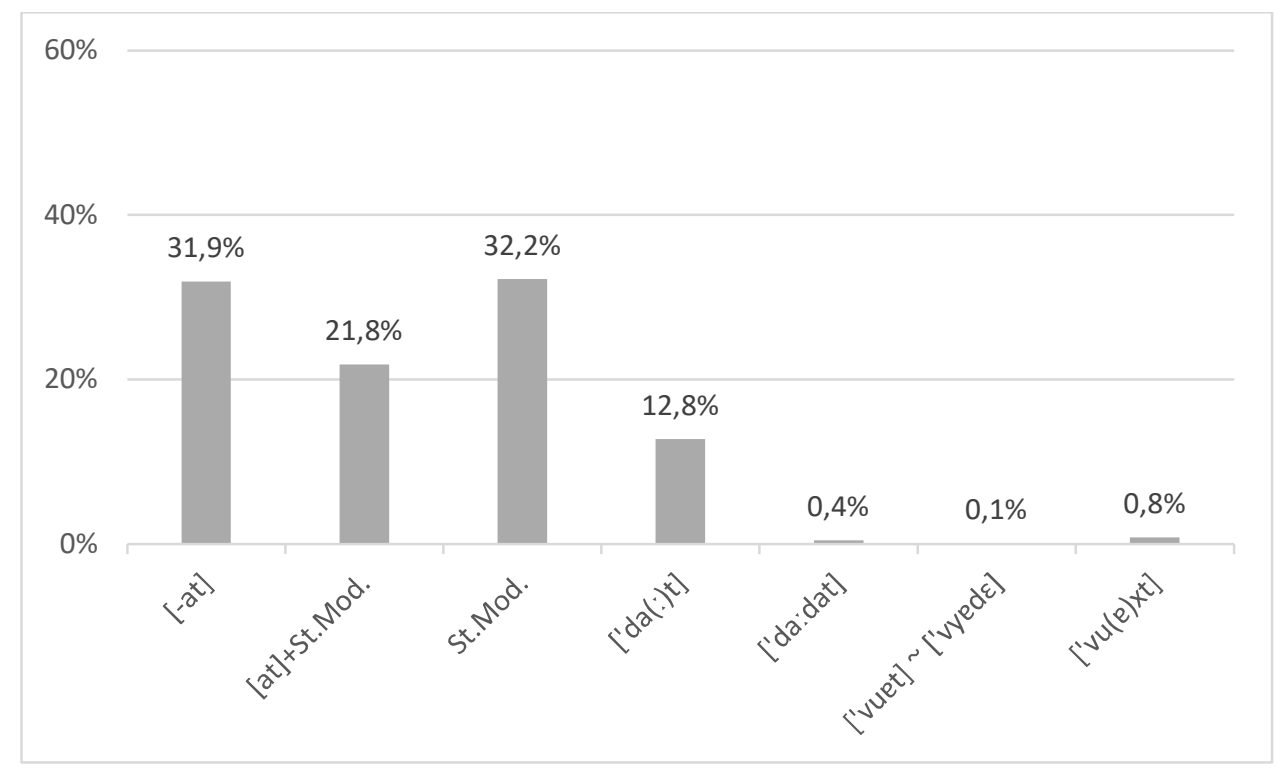

Abbildung 3: Konjunktiv-II-Bildung: gesamtes Verbalsample, Flach- und Lungau (EH I/II; n=3.136)

Insgesamt ist hier der Anteil analytischer Bildungen höher: der im gesamten Verbalsample schwach vertretene Formtyp 4 mit geringen 0,9\% (das sind ['vuet] ['vyed $\varepsilon$ ]- sowie ['vu(e)xt]Bildungen; 27 Belege) sowie Formtyp 3 mit 13,2\% (das sind die ['da(:)t] ['da:dat]-Bildungen; 414 Belege). Diesen gesamt 14,1\% analytischen Bildungen steht Formtyp 2 mit zusammen 53,7\% als dominante Gruppe gegenüber (Bildung auf -at: 1.000 Belege; 31,9\%; Bildung auf -at mit Stammmodifikation: 685 Belege; 21,8\%). Vergleichsweise stark ist auch Formtyp 1 (Stammmodifikation mit Nullmorphem) mit 1.010 Belegen (32,2\%). Deutlich wird demnach: Die synthetischen Bildungsmuster beherrschen mit 85,9\% die Morphologie des Konjunktivs II.

Für die Gruppe der starken Verben ist folgende Beobachtung noch besonderer Beachtung wert: Wie bei Mauser (1998: 349-351) festgehalten, ist bei einer Untergruppe der starken Verben eine Art Clustering erkennbar, das sich durch die aus der Ablautreihe resultierende Vokalalternanz erklärt. So sind bei starken Verben mit palatalem Vokal im Infinitiv (ggf. mit Wechsel /e/ > /i/) und zu [a] gesenktem Sekundärumlaut /ä $\sim$ æ/ im Konjunktiv II (i. e. eigentlich /a â/ im Präteritum, das allerdings in unseren Dialekten aufgrund des Präteritumschwundes nicht mehr fassbar ist) Formtypen mit Stammmodifikation viel häufiger anzutreffen als bei den starken Verben der übrigen Ablautreihen (siehe Abbildung 4). ${ }^{10}$

\footnotetext{
${ }^{10}$ Berücksichtigung fanden für diese Analyse die starken Verben aus EH I und II, um für den Lungau die Fallzahlen zu erhöhen.
} 


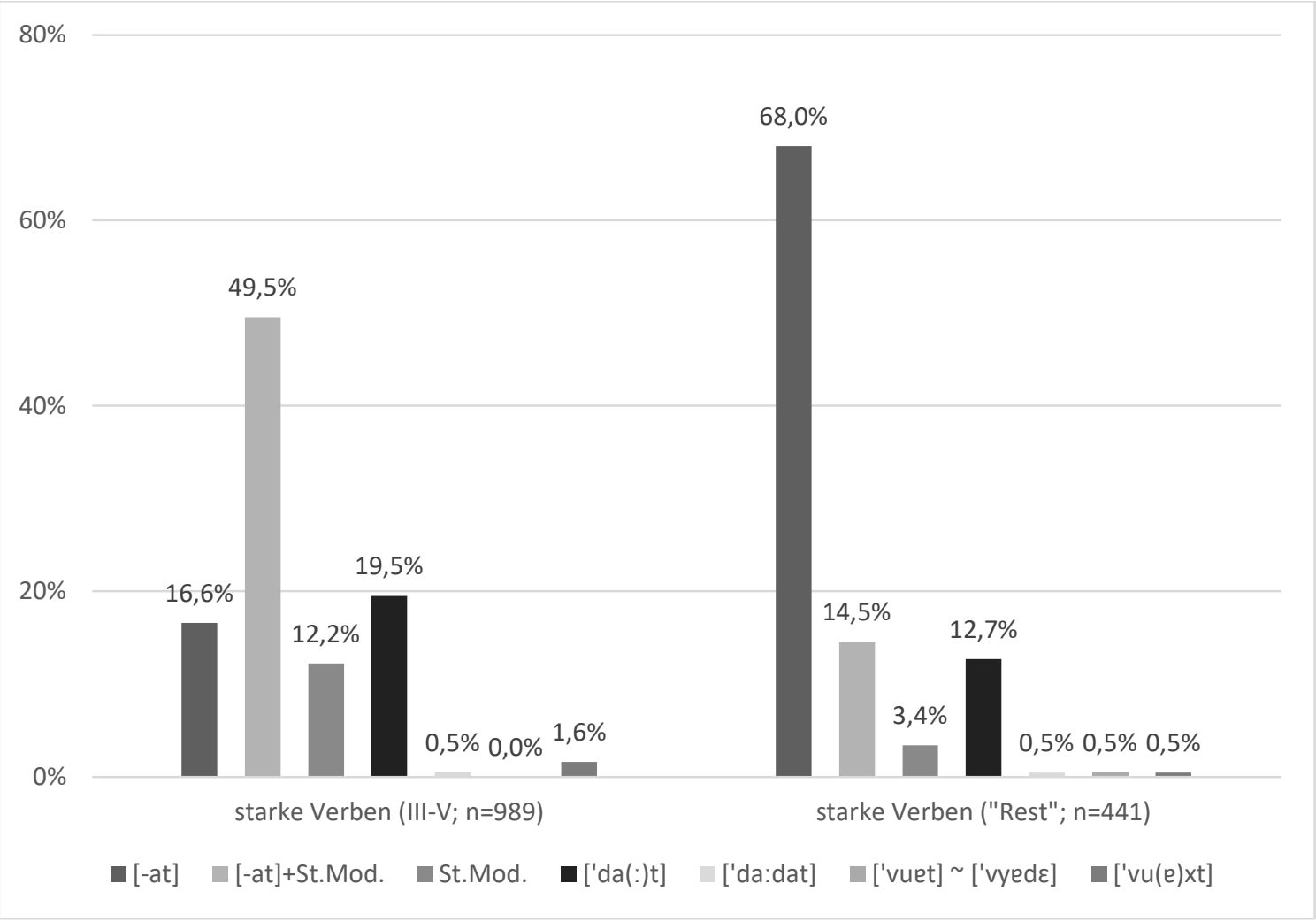

Abbildung 4: Konjunktiv-II-Bildung: Clustering starke Verben III-V, Flach- und Lungau (EH I/II)

Hier scheint sich innerhalb der starken Verben eine Klasse mit dem Merkmal Stammvokalalternanz Infinitiv /e $\sim$ i/ : Konjunktiv II /ä $\sim$ æ/ etabliert zu haben. Potenzielle Mitglieder dieser Klasse kommen regelmäßig aus den Ablautreihen III-V, das heißt Verben mit /a/ bzw. /â/ in der 2. und 3. Stammform (historische Klassen IV und V; siehe etwa Formen wie $k a(:) m$,ich/er käme' oder ga:b ,ich/er gäbe') bzw. /a/ in der 2. Stammform und sekundärer Ausdehnung auf die 3. Stammform im innerparadigmatischen Ausgleich (Klasse III; siehe etwa fant, ich/er fände $\left.{ }^{6}\right) .{ }^{11}$ Es handelt sich dabei um den Versuch der Konsolidierung einer Gruppe starker Verben im Spannungsfeld des Übergangs von starker zu schwacher Flexion - ähnlich der „Regularisierungsstrategie“, wie sie Nowak (2016) für den Standard beschrieben hat. ${ }^{12}$ In diesem Verbcluster der historischen Verbklassen III-V dominieren Konjunktiv-II-Formen mit Stammmodifikation (stammmodifizierende Nullmorphemvariante nach Formtyp 1 bzw. Morphem -at

\footnotetext{
${ }^{11}$ Cf. auch die Beobachtung von Mindl (1924/1925: 108) für Teile des oberösterreichischen Hausruckviertels, wonach Konjunktiv-II-Formen nach Formtyp 1 ,nur in der 3., 4. und 5. Ablautreihe in größerem Umfang erhalten“ wären. Selbst alle entsprechenden Beispiele, die bereits Höfer (1800: § 68) zur Illustration der „,regulären“ Bildung anführt, gehören ausnahmslos in die Ablautreihen III bis V.

12 Nowak (2016: 128) geht für den Standard von einer „,8. Ablautreihe“ aus, für die ein expandierendes Flexionsklassenmerkmal „Alternanz x-O-o (x als relativ beliebiger, meist palataler Inf.-Vokal)“ charakteristisch sei. Die Steigerung der „Generalität der Alternanzmuster“ (134) sieht sie als ein Merkmal der Regularisierungstendenz starker Verben, die entsprechende Erhöhung der Typenfrequenz führt sie unter Hinweis auf u. a. Nübling (2000) auf einen Rückgang der Tokenfrequenz zurück (Nowak 2016: 137). Vergleichbare Frequenzanalysen, wie Nowak sie durchführt (138-143), können in diesem Rahmen und v. a. auch mangels geeigneter regionalsprachlicher Korpora nicht durchgeführt werden.
} 
mit Stammmodifikation nach Formtyp 2) mit 61,8\%, während bei allen anderen starken Verben dieser Wert nur bei $17,9 \%$ liegt. ${ }^{13}$

\subsection{Regional- und generationsspezifische Variation}

Eine Gegenüberstellung der zwei politischen Bezirke (dabei ist aufgrund der Vergleichbarkeit EH II, die den Flachgau ausschloss, nicht berücksichtigt) zeigt: Aus dialektgeografischer Sicht lassen sich zwischen den beiden Regionen südmittelbairisch bzw. vorwiegend mittelbairisch geprägter Flachgau und südbairisch geprägter Lungau Unterschiede in der Konjunktiv-II-Bildung feststellen:

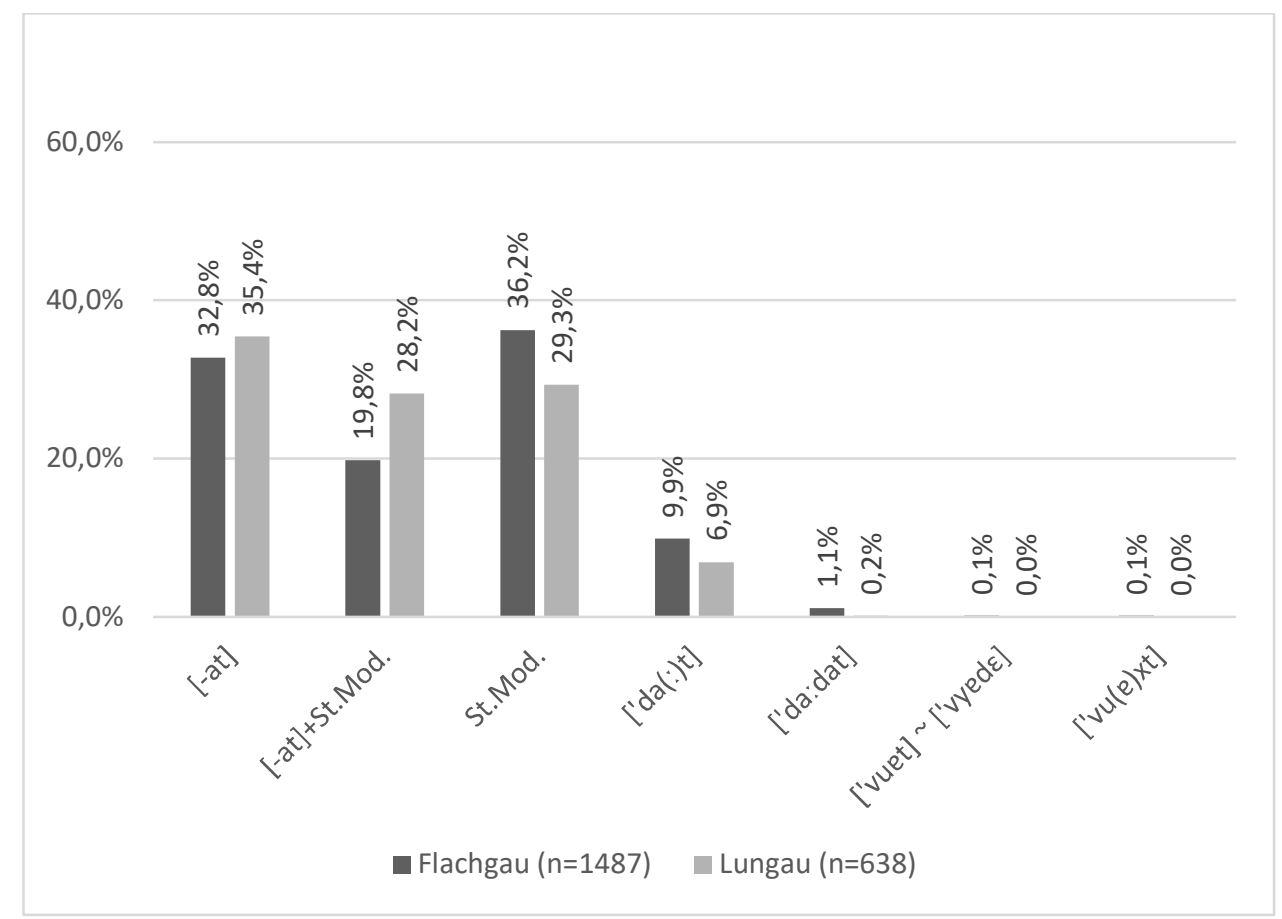

Abbildung 5: Konjunktiv-II-Bildung: gesamtes Verbsample, Flach- und Lungau getrennt (EH I)

Mit Blick auf synthetische bzw. analytische Formtypen lässt sich festhalten: Im Lungau dominieren die synthetischen Formtypen mit 92,9\% (7,1\% analytische Bildungen). Im Flachgau ist diese Dominanz etwas weniger deutlich ausgeprägt (88,8\% synthetische gegenüber $11,2 \%$ analytischen Bildungen). Die synthetische Bildung allein mit dem Morphem -at weist im Vergleich beider politischer Bezirke geringe Unterschiede auf (32,8\% im Flachgau bei 487 Belegen und $35,4 \%$ im Lungau bei 226 Belegen). Größere Unterschiede zeigen sich bei den -at-Bildungen mit Stammmodifikation: Sie sind im Lungau mit 28,2\% (180 Belege) stärker verankert als mit 19,8\% im Flachgau (294 Belege). Auffällig ist auch, dass sich im Flachgau häufiger Formen finden, bei denen der Konjunktiv II nach Formtyp 1 nur mittels Stammmodifikation gekennzeichnet ist: Im Flachgau beträgt der Prozentsatz entsprechender Formen 36,2\% (539 Belege).

\footnotetext{
13 Der Effekt dieses Clusterings ist im Flachgau, wo die stammmodifizierende Nullmorphemvariante nach Formtyp 1, wie bereits ausgeführt, stärker als im Lungau verankert ist, besonders deutlich. Hier liegen die Vergleichswerte bei 67,0\% (Stammmodifikation starke Verben Klasse III-V) gegenüber 11,6\% (Stammmodifikation starke Verben „Rest“). (Vergleichswerte Lungau: 57,3\% gegenüber 25,0\%; man beachte bei diesen Vergleichswerten allerdings, dass für den Lungau hier auch das Datenmaterial aus EH II eingeflossen ist, wodurch das Sample der starken Verben sich von dem im Flachgau etwas unterscheidet.)
} 
Im Lungau liegt dieser Wert bei 29,3\% (187 Belege). Bei den analytischen Formtypen dominiert in beiden Regionen die täte-Periphrase nach Formtyp 3, wobei der entsprechende Wert im Flachgau etwas höher ist (11,0\% aller Realisationsformen nach Formtyp 3 im Flachgau, 7,1\% im Lungau).

Vor allem um die höheren Werte für Formtyp 1 im Flachgau besser einschätzen zu können, sei ein gesonderter Blick auf die Gruppe starker Verben aus EH I geworfen, freilich ohne Berücksichtigung der Verben sein und tun: Als Verben mit suppletiven (Tempus-)Stämmen können sie kaum andere Konjunktiv-II-Formen zeigen als Formen mit Stammmodifikation (wenngleich für eine Flachgauer Gemeinde tatsächlich etwa auch eine Form ['durrat] belegt ist!).

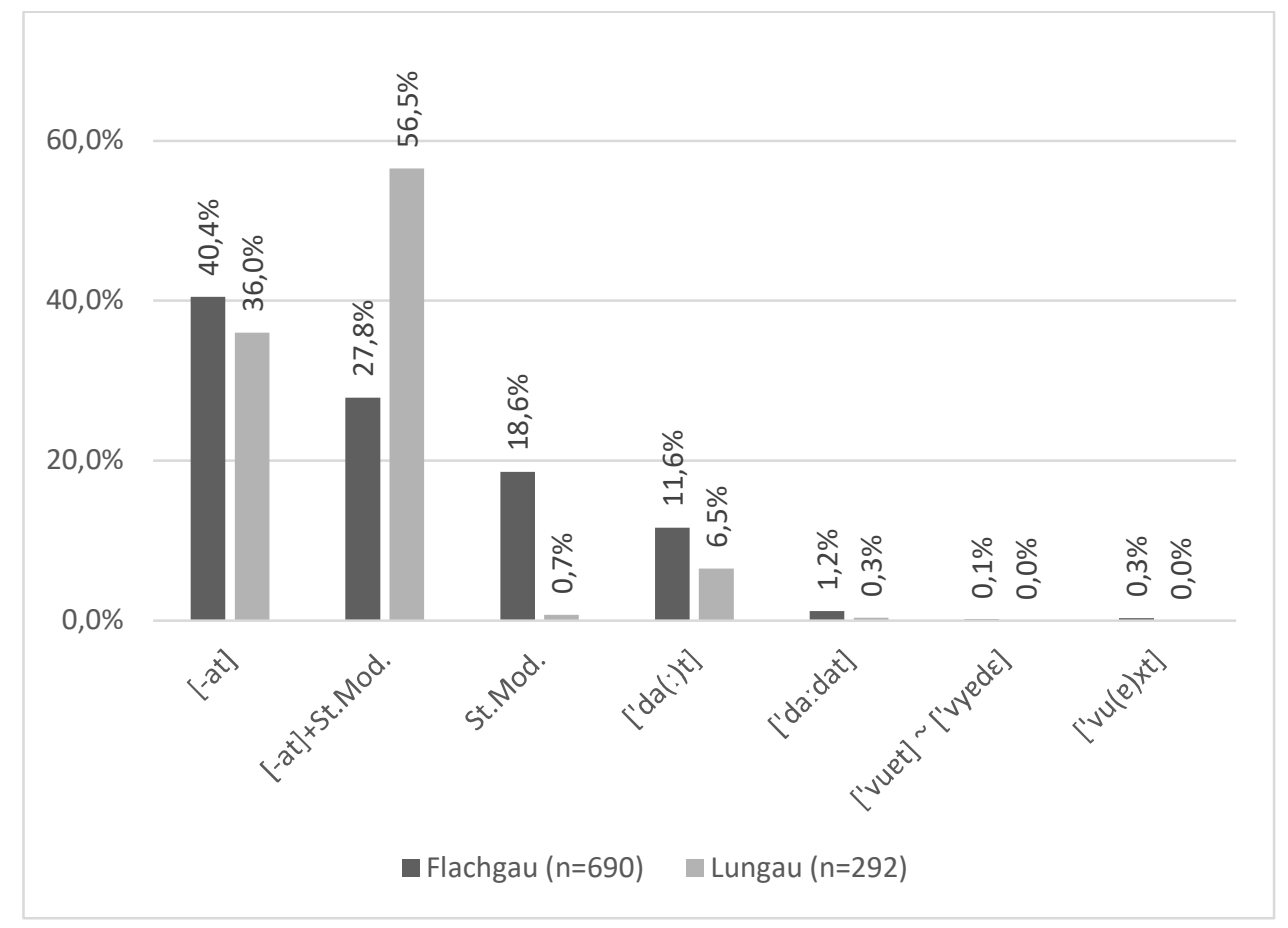

Abbildung 6: Konjunktiv-II-Bildung: starke Verben, Flach- und Lungau getrennt (EH I)

Dabei fällt auf: Der Anteil stammmodifizierender Konjunktiv-II-Formen nach Formtyp 1 ist bei den starken Verben mit 18,6\% (128 Belege; $n=690)$ im Flachgau noch deutlich höher als im Lungau, wo diese Bildung bei den starken Verben mit 0,7\% (2 Belege; n=292) als unüblich bezeichnet werden kann. Das entspricht dem bei Mauser (1998: 343-351) geschilderten Zustand, demzufolge derartige Bildungen im Lungau „Relikte einer älteren Sprachstufe“ (344) darstellen. Interessanterweise scheint sich der Formtyp 1 mit Stammmodifikation als einziger Kennzeichnung der Kategorie Konjunktiv in den (süd-)mittelbairischen Dialekten besser gegen die sprachhistorisch jüngeren Bildungsformen nach den Formtypen 2, 3 und 4 behaupten zu können. ${ }^{14}$ Dieser Trend ordnet sich nicht der gängigen Zuschreibung ,südbairisch - konservativ" unter, auch wenn man generationsbedingte Unterschiede zwischen den beiden Regionen erhebungsbedingt nicht ganz ausschließen kann.

\footnotetext{
14 Wirft man einen Blick auf die 18 Sprecher*innen aus Mittelkärnten sowie der Obersteiermark, die im Rahmen von EH 2 außerhalb des Lungaus erhoben wurden, im Rahmen dieses Beitrags aber unberücksichtigt blieben, so zeigt sich eine klare Fortsetzung der beobachteten Tendenz. Für kein einziges der erhobenen starken Verben (abgesehen von sein und tun) ist dort noch eine Konjunktiv-II-Form nur mittels Stammmodifikation belegt.
} 
Auffällig bei den starken Verben ist zudem, dass im Lungau die Konjunktiv-II-Bildung auf -at mit Stammmodifikation die dominierende Bildung darstellt (56,5\%; 165 Belege), während dieses Bildungsmuster im Flachgau deutlich schwächer ist (27,8\%; 192 Belege). Im Flachgau dominiert dagegen mit 40,4\% (279 Belege) bei den starken Verben die Bildung auf -at ohne Stammmodifikation, die im Lungau mit 36,0\% (105 Belege) vergleichsweise schwächer ist.

Nimmt man insgesamt noch die Verteilung synthetischer im Vergleich zu analytischer Kodierung in den Blick, so zeigt sich auch bei der Gruppe der starken Verben eine eindeutige Dominanz der synthetischen Kodierung: Flachgau 86,8\% : 13,2\%, Lungau 93,2\% : 6,8\%. Das Verhältnis schlägt bei den starken Verben, erwartungsgemäß, noch deutlicher zugunsten der synthetischen Kodierung aus, als dies im gesamten Verbsample schon der Fall war (Flachgau 88,8\% : 11,2\%, Lungau 92,9\% : 7,1\%). Bei den schwachen Verben (leider können, lässt man haben außer Betracht, nur die schwachen Verben nähen und lehnen ausgewertet werden) zeigt sich der gegenläufige Trend. Der Vergleichswert für beide Erhebungsgebiete zusammen beträgt dort $60,7 \%: 39,3 \%(\mathrm{n}=262)$.

Abschließend gilt die Aufmerksamkeit noch der Generationenspezifik der Formtypen im Datenmaterial, unabhängig vom Faktor Region. ${ }^{15}$ Wir erkennen in Abbildung 7, dass in allen drei Altersgruppen die synthetischen Formtypen vorherrschen, in der Altersgruppe der 20- bis 39Jährigen mit 86,0\%, bei den 40- bis 59-Jährigen mit 92,1\% und bei der Gruppe der Generation 60+ mit 90,2\%. Umgekehrt formuliert: Der Anteil analytischer Formtypen ist in der Altersgruppe 20-39 mit 14,0\% am höchsten. In der Altersgruppe 40-59 liegt der Wert deutlich niedriger bei 7,9\%, in der Generation 60+ mit 9,8\% wieder etwas höher. (Dass hier der Trend nicht völlig linear verläuft, hat wahrscheinlich mit den geringen Fallzahlen in der Generation $60+\mathrm{zu}$ tun.) Der vergleichsweise hohe Prozentsatz analytischer Formtypen in der Altersgruppe der 20bis 39-Jährigen kommt im Wesentlichen durch die häufigere Verwendung des Formtyps 3 (tätePeriphrase $-\left[{ }^{\prime} \mathrm{da}(:) \mathrm{t}\right] \sim\left[{ }^{\circ} \mathrm{da}:\right.$ dat $]+$ Infinitiv) zustande.

\footnotetext{
15 Wie in Fußnote 9 bereits kurz festgehalten, wurde bei der generationenspezifischen Auswertung nur das Datenmaterial aus EH I berücksichtigt, da es Datensätze sowohl aus dem Flach- als auch aus dem Lungau umfasst. Die Lungauer Datensätze aus EH II wurden nicht zu den Lungauer Datensätzen von EH I geschlagen: Ein Einfluss der 12 bis 18 Jahre Echtzeitdiachronie zwischen den beiden Erhebungen kann nicht ausgeschlossen werden.
} 


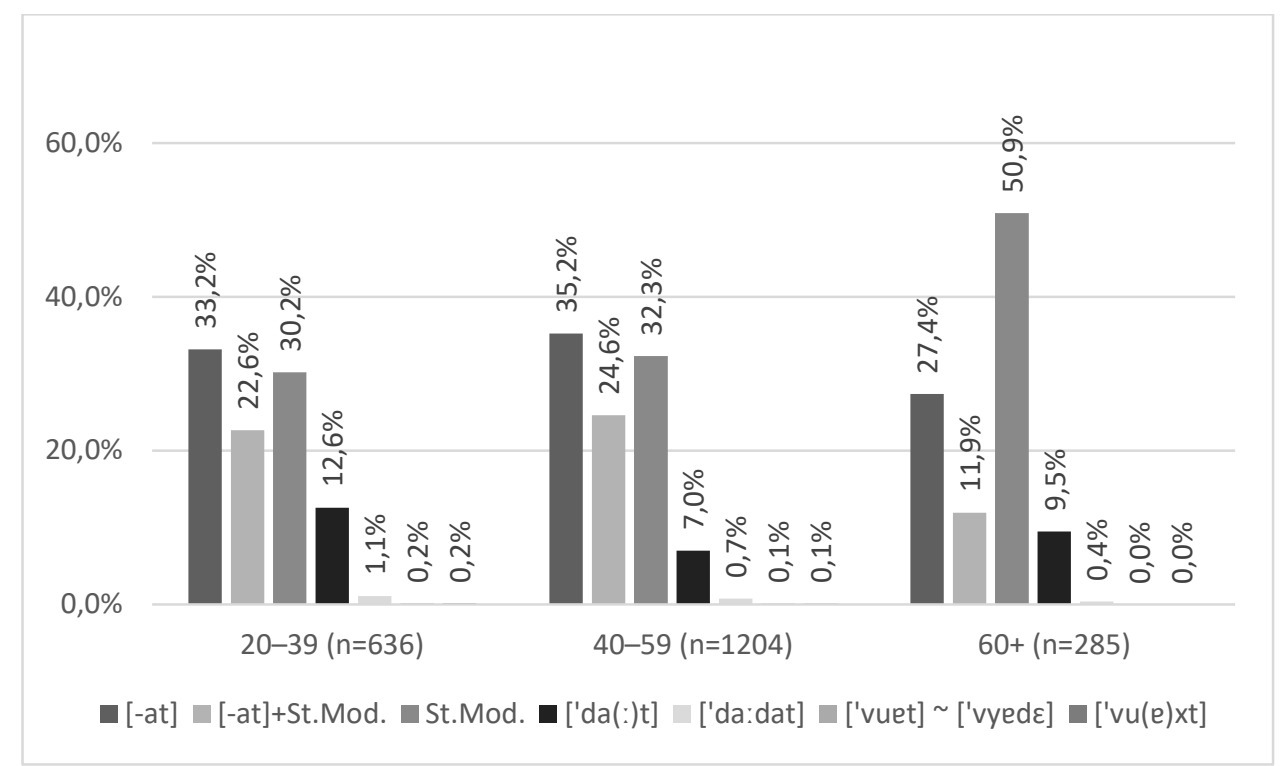

Abbildung 7: Konjunktiv-II-Bildung nach Altersgruppen (EH I)

\subsection{Zusammenfassung der dialektologischen Befunde}

Die Auswertung der dialektologischen Daten zeigt für das Sample aller Verben (stark, schwach, modal) eine starke Dominanz der synthetischen $(85,9 \%)$ im Vergleich zu analytischen Formtypen (14,1\%). Die höchste Gebrauchsfrequenz der analytischen Formtypen findet man in der jüngsten Altersgruppe (20-39 Jahre). Die Dominanz der synthetischen Formtypen ist für die Modalverben noch deutlicher (93,9\% synthetische Formtypen). Nur 6,1\% entfallen auf die analytische Kodierung, genauer auf den Formtyp 3 (täte-Periphrase). Der Formtyp 4 (würde-Periphrase) spielt hier, wie auch im gesamten Verbsample, keine Rolle.

Aus flexionsmorphologischer Sicht bemerkenswert ist die Beobachtung, dass sich innerhalb der starken Verben eine Subklasse zu etablieren scheint, in der die Modifikation des Stammsilbenvokals von /e $\sim \mathrm{i} /$ im Infinitiv zu /ä $\sim$ æ/ im Präteritum (Stammform für den Konjunktiv II) als zentrales Merkmal der Flexionsklassenzugehörigkeit fungiert.

\section{Online-Umfrage}

\subsection{Datengrundlage}

Unsere Datenquelle ist die erste Runde einer großen Online-Umfrage per LimeSurvey, die im September und Oktober 2019 in Kooperation mit der Tageszeitung Salzburger Nachrichten (SN) beworben und verteilt wurde ${ }^{16}$ (cf. ähnlich im Ansatz auch Leemann/Derungs/Elspaß 2019). Die Kooperation mit diesem im Bundesland dominanten Medium bot die Aussicht auf einen generell starken und regional eng gerasterten Rücklauf: Die Print-SN hatten im Jahr 2019/20 eine relative Reichweite im Bundesland (gegenüber anderen Printmedien) von nicht weniger als 31,7\% (cf. Media-Analyse 2019/20), das Online-Angebot wies 2018 ca. 4,5 Mio.

\footnotetext{
16 Die Methodik wurde bereits im Sommersemester 2019 erfolgreich von Konstantin Niehaus für das Bundesland Tirol in Kooperation mit der Tiroler Tageszeitung in einem Pilotprojekt („Atlas zur tirolerischen Alltagssprache“) getestet: Im Rahmen eines Projektseminars wurden für Tirol die ersten 10 AdA-Runden in angepasster Form von Studierenden mittels LimeSurvey-Fragebogen wiederholt.
} 
Visits und ca. 1,5 Mio. Unique Clients auf, die mobilen Angebote hatten im selben Zeitraum ca. 2,8 Mio. Visits und 850.000 Unique Clients (cf. ÖWA 2018). Die Proband*innenauswahl ist bei diesem Ansatz zwar geringer kontrollierbar als bei Ortserhebungen, bietet aber eine niedrigere Einstiegsschwelle in die Teilnahme und verringert durch den anonymen Zugang das Problem, selbstbewusste (lokalpatriotische) oder (im anderen Extremfall) Satisficing ${ }^{17}$ betreibende Proband*innen womöglich bevorzugen zu müssen (dass alle Proband*innen dennoch überhaupt an regionaler oder lokaler Sprache interessiert sein werden, bleibt konstant).

Die Umfrage fand in insgesamt vier Runden (eine pro Woche) statt, wobei alle Kanäle der SN - print, online und (online) mobil (v. a. Facebook-Page) - bespielt wurden. Die Rundeninhalte basieren auf der Item- und Fragestruktur des Atlas zur deutschen Alltagssprache (AdA) (Elspaß/Möller 2003-), wurden aber methodisch für die Kooperation und inhaltlich für Salzburg - etwa durch die Abfrage auch traditioneller Dialektvarianten - angepasst. Anders als im AdA wurde außerdem nicht nach ortsüblichen Varianten gefragt, sondern - eingedenk möglicher Mobilität der Sprecher*innen und zu vermeidender Vorannahmen zur regionalen Sprecher*innenidentität und Authentizität (cf. zur Theorie dieses Aspekts Niehaus 2021) ${ }^{18}$ - explizit nach der persönlich üblichen Variante. Der wohl größte Schritt bestand jedoch darin, die Umfragerunden des AdA zugunsten einer populären Präsentation der Umfrage (z. B. über die Facebook-Page der SN) und einer Erhöhung des Spielfaktors rigoros zu kürzen. ${ }^{19}$ Aus den gewonnenen Daten wird ein Atlas zur Salzburger Alltagssprache (ASA) in Buchform entstehen. ${ }^{20}$

Die Umfrage brachte insbesondere zu Beginn einen massiven Rücklauf, ${ }^{21}$ der unsere Erwartungen sogar noch übertraf und ab der zweiten Runde relativ stabil blieb. ${ }^{22}$ Die erste Runde erreichte nach grober Schätzung 1,5\% bis $2 \%$ der gesamten Salzburger Bevölkerung. ${ }^{23}$

Unsere für die aktuelle Auswertung zum Konjunktiv II relevanten Daten stammen aus Runde 1 der Erhebung. In die hier präsentierte Analyse flossen nur Datensätze ein, die eine Antwort auf die Konjunktiv-Frage (Frage 12) enthielten sowie eindeutig einem Ort im Bundesland Salzburg zuordenbar waren. Dies führte zu einem letztlich gültigen Set von 4.526 auswertbaren Datensätzen. 1.453 Datensätze stammen dabei von männlichen Teilnehmern, 3.012 Datensätze von weiblichen $($ Rest = keine Angabe). Die Verteilung auf Alters- und Bildungsgruppen sowie

\footnotetext{
${ }^{17}$ Satisficing (aus satisfying und sufficing) meint das Verhalten, nur (noch) „beiläufig“ und ohne „positive Gründe“ teilzunehmen; dies kann sich auch erst im Laufe der Teilnahme ergeben (cf. Moosbrugger/Brandt 2020: 79).

18 Auch Erfahrungen aus dem Pilotprojekt flossen ein, etwa dass laut rückgemeldeter Kommentare trotz anderslautendem Wunsch nicht die ortsüblichen, sondern die eigenen Varianten angeklickt wurden (weil die unpersönliche Fragestellung als unnatürlich beurteilt oder schlicht im Laufe der Umfrage vergessen wurde).

${ }^{19}$ Im Pilotprojekt erwies es sich bereits als förderlich für den Rücklauf, die Fragenanzahl unter 30 (inkl. Abfrage der Metadaten) bzw. 20 (exkl. Abfrage der Metadaten) zu halten.

${ }^{20}$ Der ASA wird vom Land Salzburg gefördert (Referat 02/04: Kultur und Wissenschaft, Laufzeit: 21.12.2020 31.07.2023).

${ }^{21}$ Mehrfachbearbeitungen und willentliche Falschbearbeitungen (,trolling ${ }^{\star}$ ) sind zwar theoretisch möglich, beides erschien aber auch in ähnlichen (u. a. thematisch kaum politisierten oder ideologisierten), crowd sourcing'-Erhebungen unwahrscheinlich (cf. Leemann/Derungs/Elspaß 2019: 24).

${ }^{22}$ Rücklauf insgesamt (inkl. unbereinigter und unvollständiger Datensätze): Runde 1: $\mathrm{n}=9.655$; Runde 2: $\mathrm{n}=3.509$; Runde 3: $\mathrm{n}=3.089$; Runde 4: $\mathrm{n}=2.850$.

${ }^{23}$ Gemessen an ca. 9.100 Aufrufen des Fragebogens gegenüber ca. 560.000 Bewohner*innen.
} 
die Salzburger Bezirke kann Tabelle 2 entnommen werden. Unter den Angaben zum Bildungsgrad fällt die hohe Anzahl an Studiums- und Doktoratsabschlüssen auf, die jedoch aufgrund der Erhebungssituation (hierzu zählt u. a. das Vorhandensein einer Universität im Gebiet) erwartbar war und während der Auswertung gewichtet werden kann.

\begin{tabular}{|l|l|}
\hline Altersgruppen & $\mathbf{n}$ \\
\hline 0-19 Jahre & 312 \\
\hline 20-39 Jahre & 2.449 \\
\hline 40-59 Jahre & 1.159 \\
\hline 60+ Jahre & 540 \\
\hline & \\
& \\
& \\
\hline
\end{tabular}

\begin{tabular}{|l|l|}
\hline Bildungsgruppen & n \\
\hline (noch) kein Abschluss & 35 \\
\hline Pflichtschulabschluss & 623 \\
\hline $\begin{array}{l}\text { Fach-/ } \\
\text { Handelsschulabschluss }\end{array}$ & 675 \\
\hline Matura & 1.412 \\
\hline Studiumsabschluss & 1.502 \\
\hline Doktorat & 201 \\
\hline
\end{tabular}

\begin{tabular}{|l|l|}
\hline Bezirke & n \\
\hline Stadt Salzburg & 1.498 \\
\hline $\begin{array}{l}\text { Salzburg Umgebung } \\
\text { (Flachgau) }\end{array}$ & 1.351 \\
\hline $\begin{array}{l}\text { Hallein (Tennen- } \\
\text { gau) }\end{array}$ & 440 \\
\hline St. Johann (Pongau) & 511 \\
\hline $\begin{array}{l}\text { Zell am See (Pinz- } \\
\text { gau) }\end{array}$ & 526 \\
\hline Tamsweg (Lungau) & 200 \\
\hline
\end{tabular}

Tabelle 2: Hintergrunddaten der Teilnehmer/innen

Die Frage und Antwortvorgaben zum Konjunktiv II, die vorrangig auf morphologische Varianten abzielten, lauteten wie folgt (ohne Möglichkeit der Mehrfachnennung ${ }^{24}$ ):

Zwei Kinder versuchen vergeblich, auf einen Baum zu steigen, in dem ihr verlorener Ball steckt. Sie geben auf und meinen: „Da ... wir eine Leiter ...“(Aber es ist keine da.)

- bräuchten

- täten/dadn/dadadn ... brauchen

- braucherten/brauchatn

- bräucherten/bräuchatn

- würden ... brauchen

- keine Antwort

Da es sich um eine Erhebung zur Alltagssprache handelt, wurde die im bairisch-österreichischen Raum eher unübliche (cf. Glauninger 2011a: 101) und zudem ambige (cf. Hennig 2016: 180) Form brauchten nicht erhoben. Die Verschriftung der Antwortmöglichkeiten versteht sich außerdem im Sinne der gebotenen Simplizität und Übersichtlichkeit bei Frageitems zur Syntax nur als grobe Annäherung. So wurde z. B. nicht weiter unterschieden nach apokopierten, klitisierten oder Allegro-Formen, und auch nicht nach dialektnäheren Lautungen, sondern die Auskunftspersonen wurden gebeten, sich die Variante in ihrer eigenen Aussprache vorzustellen. Aussprachevarianten (z. B. entrundete Formen) von brauchen wurden somit nicht angegeben, allerdings geschah dies auch, um den Fokus der Teilnehmer*innen auf die Morphologie zu lenken (einige Proband*innen nannten ansonsten phonetisch motivierte Korrekturschreibungen im Freitextfeld am Ende des Fragebogens, diese sind jedoch quantitativ vernachlässigbar).

\footnotetext{
${ }^{24}$ Dies erhöht zwar den Bias auf frequente Varianten, wurde aber bei diesem Item aus zweierlei Gründen in Kauf genommen: Zum einen erhöht einfaches Klicken im Sinne der Spontaneität die Bearbeitungsgeschwindigkeit und -motivation der Teilnehmer*innen, zum anderen bevorzugen Sprecher*innen unter mehreren Varianten i. d. R. in Bezug auf eine bestimmte Situation (wie hier angezielt) eine bestimmte. Für Proband*innen, die hier mehrere Varianten nennen wollten, gab es die Möglichkeit zur freien Nennung am Ende des Fragebogens, dortige Angaben wurden in der folgenden Analyse berücksichtigt.
} 
Trotz dieser Einschränkungen ist davon auszugehen, dass bräuchten am ehesten eine Standardvariante darstellt, braucherten/brauchatn hingegen eindeutig dialektal einzuordnen ist, während der vertikale Status der übrigen Varianten aktuell nicht vollständig zu klären sein wird. Unseres Wissens bislang in der Forschung nicht in Betracht gezogen wurde bräucherten/bräuchatn, ein Variantentyp, der morphologisch betrachtet dialektuntypisch Umlaut und -at kombiniert, und zum ersten Mal umfassend abgefragt wurde. Durch die Wahl des Verbs brauchen kann Typ 1 (stammmodifizierende Nullmorphemvariante) in die Analyse nicht einbezogen werden; dies tut jedoch der Veranschaulichung genereller Tendenzen keinen Abbruch.

\subsection{Hypothesen}

Auf der Basis der in Abschnitt (2) angeführten Studien sind aus variationslinguistischer Sicht mindestens folgende außersprachliche Faktoren, die für die Wahl der Konjunktivform mitentscheidend sein können, festzuhalten: Alter, Infrastruktur bzw. landschaftlicher Charakter des Wohngebiets (rural vs. urban) und Mobilität der Sprecher*innen sowie Standard-Orientierung der Sprecher*innen. Auf der Ebene der Konjunktivrealisierungen kann generell zwischen analytischen und synthetischen Formen differenziert werden, und zudem können die Realisierungen hinsichtlich ihrer (strukturellen und/oder pragmatischen) Position auf dem Dialekt-Standard-Spektrum eingeordnet werden. Insgesamt lassen sich folgende Hypothesen formulieren:

- Hypothese A: Über alle Variationsfaktoren hinweg zeigt sich eine Präferenz für die synthetische Form mit -ad/-at (siehe Ergebnisse oben, 3.2).

- Hypothese B: Wenn eine analytische Konstruktion verwendet wird, dann ist dies mehrheitlich der täte-Konjunktiv (siehe Ergebnisse oben, 3.2).

- Hypothese C: Je jünger der/die Sprecher*in ist, desto eher wird eine analytische Konstruktion verwendet (cf. Breuer/Wittibschlager 2020 und Ergebnisse oben, 3.3).

- Hypothese D: Standardformen und standardnahe Formen sind eher im urbanen Raum als auf dem Land anzutreffen (cf. ibd.; Lenz 2019).

- Hypothese E: Je länger durch bildungsinstitutionelle Anforderungen Standarddeutsch von Sprecher*innen verlangt worden ist (gemessen am formalen Bildungsabschluss), desto häufiger verwenden Sprecher*innen die Standardformen (cf. Steinegger 1998: 153-167).

- Hypothese F: Je höher die Mobilität von Sprecher*innen ist, desto eher verwenden sie die Standardformen (cf. Steinegger 1998: 300-301).

Da bislang keine spezifischen Studien zur geografischen Verteilung der Konjunktiv-II-Typen im gesamten Land Salzburg existieren, gehen wir zunächst - auch unter dem Eindruck der dialektologischen Ergebnisse, siehe 3.3 - von Unterschieden zwischen den Dialekträumen und Verwaltungseinheiten (Bezirken) aus.

- Hypothese G: Es gibt Unterschiede in der Häufigkeitsverteilung der Formtypen zwischen den Dialekträumen und Verwaltungseinheiten (Bezirken).

\subsection{Ergebnisse Hypothesen A und B}

Betrachten wir zunächst die Konjunktiv-II-Varianten in ihren generellen Häufigkeiten laut Online-Umfrage und damit Hypothese A (Tabelle 3). Wie erwartet präferierten die Befragten für 
die Salzburger Alltagssprache mit großer Mehrheit (66,5\% der Antworten) die dialektalen Formen braucherten/brauchatn (Formtyp 2). Formtyp 3, der täte-Konjunktiv, erreicht ca. 15\%. Auf Basis der Ergebnisse von Breuer/Wittibschlager (2020: 153-154) überraschend, jedoch die dialektologischen Befunde aus Abschnitt (3) bestätigend, wird offenbar die würde-Variante (Formtyp 4) äußerst wenig verwendet - überhaupt erreichen analytische Formen nicht mehr als knappe 20\%. In diesem Zusammenhang lässt sich Hypothese B eindrucksvoll bestätigen, also, dass täte-Konjunktive eher verwendet werden als würde-Konjunktive (sogar die synthetische Standardvariante bräuchten erreicht fast doppelt so viele Nennungen wie Formtyp 4).

\begin{tabular}{|l|l|l|}
\hline Variante & n & Prozent \\
\hline bräucherten/bräuchatn & 331 & 7,3 \\
\hline bräuchten & 337 & 7,4 \\
\hline braucherten/brauchatn & 3.010 & 66,5 \\
\hline täten/dadn/dadadn ... brauchen & 674 & 14,9 \\
\hline würden ... brauchen & 174 & 3,8 \\
\hline Gesamt & $\mathbf{4 . 5 2 6}$ & $\mathbf{1 0 0 , 0}$ \\
\hline
\end{tabular}

Tabelle 3: Generelle Häufigkeiten der Antworten bei Runde 1, Frage 12

Unerwartet ist die generell hohe Zahl an Angaben für bräucherten/bräuchatn - dieses Ergebnis kann kaum mehr plausibel über methodische Bias-Effekte, z. B. über die versehentliche Auswahl der Variante, Reihenfolgeeffekte (der einzelnen Antwortmöglichkeiten), soziale Erwünschtheit oder Satisficing erklärt werden. Vielmehr könnte es sein, dass manche Sprecher*innen die Endung -at gewissermaßen als Default-Dialektmarker übergeneralisieren und an die eigentlich im Bairischen unüblichen umgelauteten Formen anhängen. Die Übergeneralisierung würde noch zusätzlich dadurch begünstigt, dass beim Formtyp 2 ja tatsächlich in vielen Fällen eine Stammvokalmodifikation eintritt (bedingt durch Ablaut, nicht Umlaut - doch dies kann möglicherweise von Sprecher*innen nicht systematisch unterschieden werden). Dass die Form prinzipiell möglich ist, zeigen zudem manche Kommentare, die die Form affirmativ wiederholen, „auch nur ,bräuchat ${ }^{\text {‘ }}$ - verwende beides [sic]“ (ID 902, 56-jährige Angestellte aus Salzburg), ,bräuchat (ohne n), ,ma‘ statt ,wir““( (ID 7543, 31-Jähriger im öffentlichen Dienst in Lehre/Forschung aus Salzburg).

\subsection{Ergebnisse Hypothesen $\mathrm{C}$ bis $\mathrm{G}$}

Wir beginnen mit der breit angelegten Hypothese G, für die sich insgesamt eine gemischte Evidenz ergibt. Die Annahme einer regionalen Verteilung haben wir operationalisiert, indem Dialektregionen im Bundesland Salzburg und Bezirksgrenzen als Faktoren (in Chi-QuadratTests) getestet wurden. Als Dialektregionen wurden nach gängigen Klassifizierungen (cf. Reiffenstein 1980; Lenz 2019; Mauser 2021a) das Westmittelbairische (nördlicher Flachgau), das Südmittelbairische (südlicher Flachgau inkl. Stadt Salzburg, Bezirke Hallein, St. Johann sowie Zell am See) und das Südbairische (Bezirk Tamsweg) angenommen. ${ }^{25}$ Bei der Analyse deutet sich eine Sonderstellung der Stadt Salzburg an: Während sich bei Einbezug der Stadt Salzburg

\footnotetext{
${ }^{25}$ Zwei als „Übergangsgebiete“ definierte Regionen - eine entlang einer Ost-West-Linie im Flachgau zwischen West- und Südmittelbairisch, eine andere im südwestlichen Pinzgau zwischen Südmittel- und Südbairisch (,Tirolerisch") - erwiesen sich als statistisch irrelevant.
} 
in das Südmittelbairische signifikante Unterschiede zwischen den Dialektgebieten abzeichnen, verlieren die Unterschiede an Signifikanz und Effektstärke, wenn man die Stadt Salzburg ausklammert. Deskriptiv hebt sich dann noch am ehesten das Südbairische mit mehr brauchertenFormen und weniger täten-Konjunktiven von den anderen beiden Regionen ab, während das Westmittelbairische und das Südmittelbairische bemerkenswert ähnlich sind.

Eine geografisch stärker differenzierbare signifikant unterschiedliche Verteilung der Varianten zeigt sich hingegen bei der Analyse nach Salzburger Bezirken $\left(\chi^{2}(20)=351,14\right.$; $p<, 001$; Cramér's V =0,139) (siehe Abbildung 8). Paarweise Vergleiche (siehe Anhang A1) zeigen, dass sich vor allem die Stadt Salzburg von allen anderen Bezirken abhebt. Aber auch zwischen den anderen Bezirken gibt es teilweise statistisch signifikante Unterschiede. Ein Blick auf die relativen Häufigkeiten und die standardisierten Residuen (siehe Anhang A2) erlaubt genauere Aussagen zu den entscheidenden Unterschieden: So verstärkt sich die Tendenz zum Formtyp 2 (mit -at-Suffix) in den südlicheren Bezirken Tamsweg (Lungau) und Sankt Johann (Pongau) (80\% bzw. 79,8\%). Anders stellt sich die Lage im westlichsten Bezirk des Bundeslandes, Zell am See (Pinzgau), dar: Die Tendenz zum Formtyp 2 ist zwar auch hier ausgeprägt, daneben jedoch überrascht die im Vergleich der Bezirke höchste Frequenz des täte-Konjunktivs (Formtyp 3). Es besteht jedoch hier eine leichte Korrelation mit dem Alter, die diese Häufigkeitsverteilung teilweise erklären kann (siehe unten): Tatsächlich weisen die Rückmeldungen aus dem Pinzgau einen hohen Anteil jüngerer Proband*innen bzw. sogar unter allen Bezirken den höchsten Anteil der jüngsten Proband*innengruppe ( 0 bis 19 Jahre) auf ( 87 von $521=16,7 \%$ ), allerdings bleibt die vergleichsweise höhere Tendenz zu täte-Formen im Pinzgau auch in Gruppen mit höherem Alter erhalten (23,9\% bei den 20-39-Jährigen; 15,4\% bei den 40-59-Jährigen; 7,8\% bei den 60+-Jährigen).

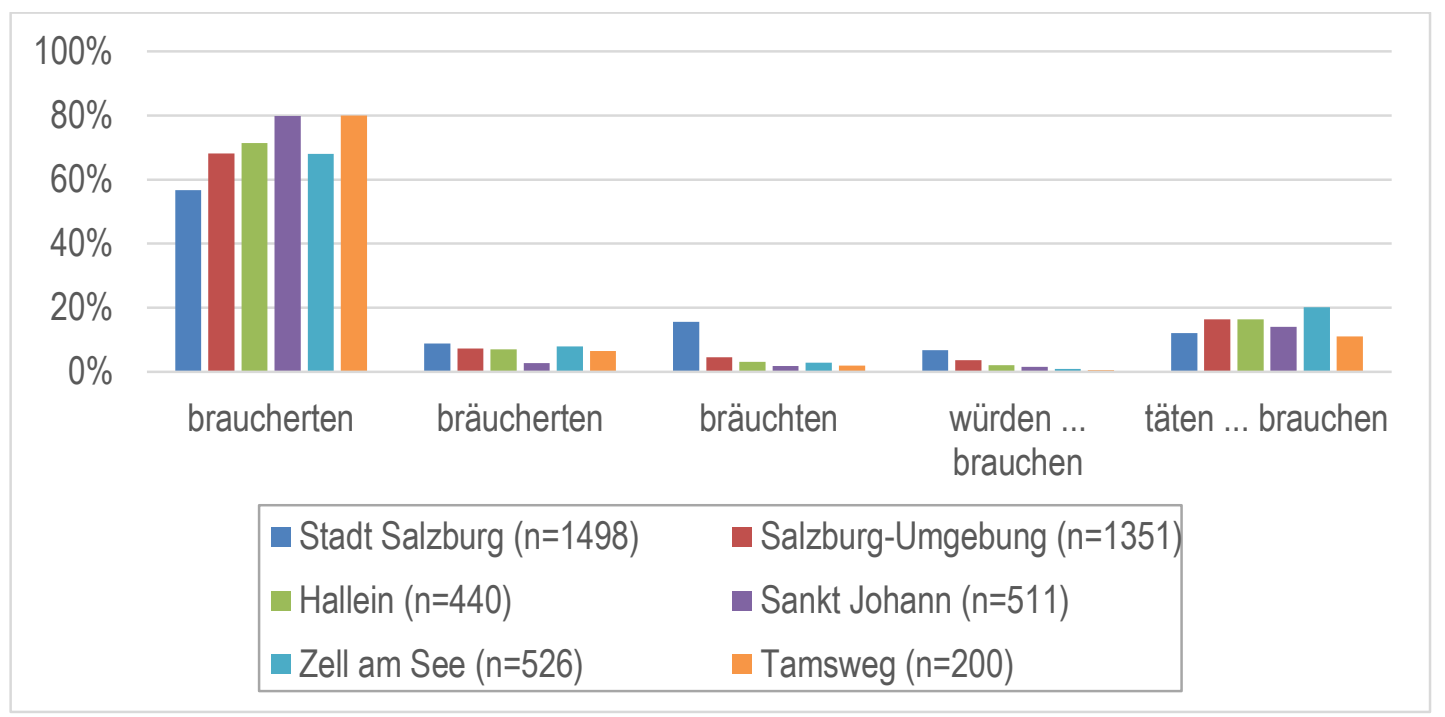

Abbildung 8: Regionale Verteilung der Konjunktiv-II-Varianten von brauchen auf Bezirksebene

Hypothese D lässt sich nun in diesem Zuge ebenfalls bereits vorläufig bestätigen: Erwartungsgemäß sind die Nennungen für die Standardvarianten bräuchten und würde-Konjunktiv (Formtyp 4) im Stadtbezirk am höchsten. Zur weiteren Prüfung der Hypothese D unter Einbezug eines größeren Agglomerationsgebiets rund um die Stadt Salzburg wurden die Flach- und Tennengauer Gemeinden nach Nähe zur Stadt Salzburg (binär) kategorisiert. Stadtnähe wurde 
dabei infrastrukturell definiert als Zugehörigkeit des jeweiligen Ortskerns zum Liniennetzplan „Region Salzburg Stadt“ des Salzburger Verkehrsverbundes (cf. Salzburg Verkehr 2021). Die Gegenüberstellung der stadtnahen und stadtfernen Gemeinden aus diesen beiden Bezirken bestätigt die statistisch signifikanten Unterschiede $\left(\chi^{2}(4)=27,317 ; p<, 001\right.$; Cramér's V $\left.=0,124\right)$, die sich vor allem in einer Reduktion der braucherten-Variante und einer höheren Frequenz der Standardvarianten in den stadtnahen Gemeinden niederschlagen.

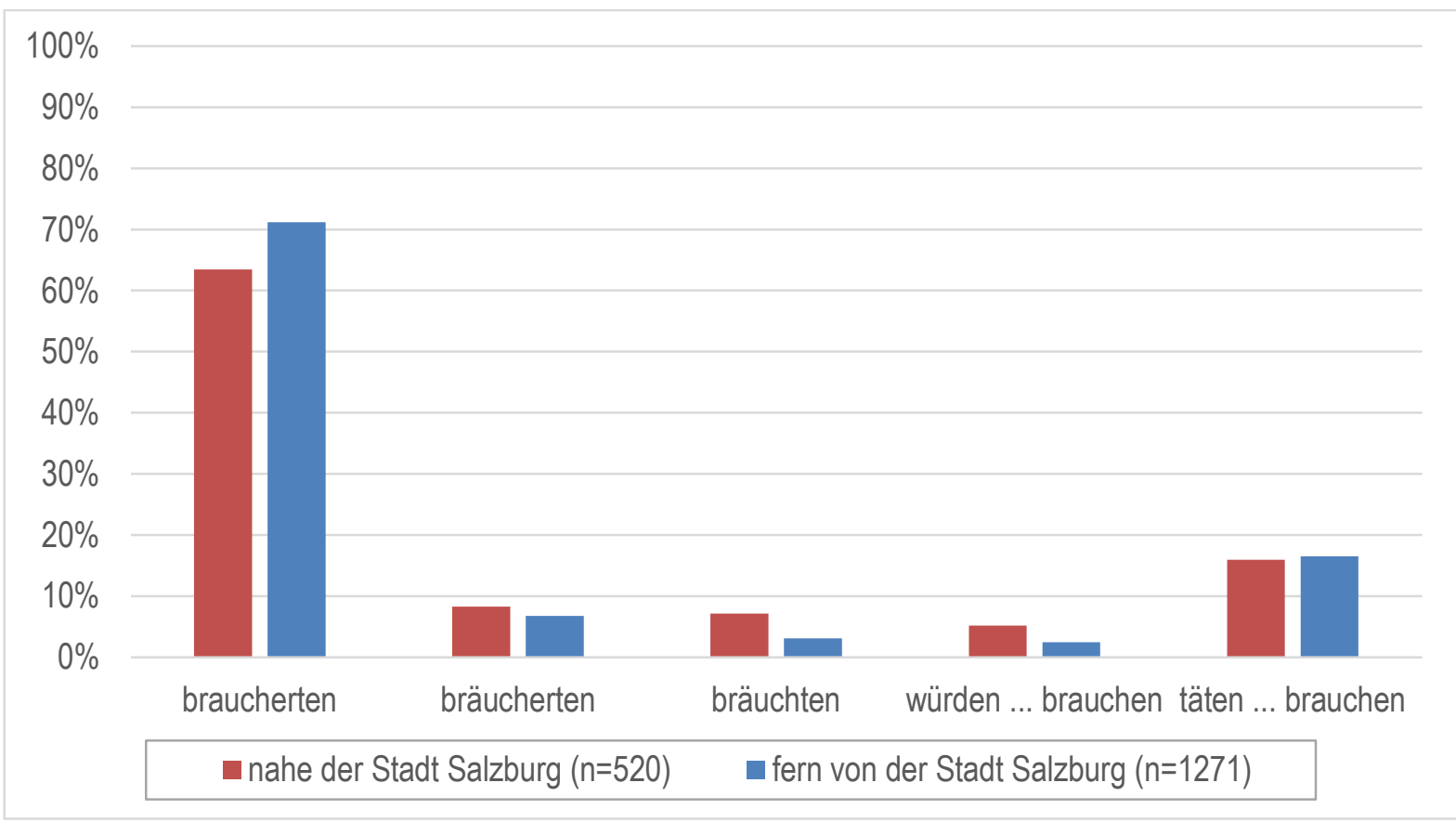

Abbildung 9: Regionale Verteilung der Konjunktiv-II-Varianten von brauchen in den Bezirken Salzburg-Umgebung und Hallein nach Nähe zur Stadt Salzburg

Es liegt nahe, diese Verteilung der Standardvarianten als Abbild der gestiegenen Mobilität und auch der neuerlichen Landflucht städtischer Sprecher*innen ob hoher Wohnkosten in der Landeshauptstadt zu sehen (cf. hierzu auch „counterurbanisation“, Britain 2012).

Um schließlich zu überprüfen, ob möglicherweise auch andere regionale Zentren ähnliche Wirkung entfalten wie die Stadt Salzburg, wurde die Gemeindegröße als Variable berücksichtigt. Diese zeigte jedoch unter Ausklammerung der Stadt Salzburg bei einer groben dreistufigen Klassifizierung der Gemeinden als klein (unter 5.000 Einwohner), mittel (5.000-10.000 Einwohner) und groß (über 10.000 Einwohner) keinen statistisch signifikanten Zusammenhang mit der Verteilung der Varianten.

Immerhin erlauben die Überprüfung der Hypothese D sowie die Messung der Stadtnähe schließlich eine nähere Einordnung der Variante bräucherten/bräuchatn und des täte-Konjunktivs nach Standard(-nähe) oder Dialekt(-nähe): Die Variante bräucherten/bräuchatn wird mit leichter Tendenz eher in stärker standardaffinen Gebieten gewählt, sodass sie als standardnähere Variante gesehen werden kann (und damit tatsächlich eher bräucherten, nicht braicherten o. ä. lautet). Der täte-Konjunktiv verteilt sich in unseren Daten regional hingegen eher wie die 
dialektale Variante braucherten/brauchatn (Typ 2). Das spricht dafür, dass von vielen Proband*innen im Bundesland Salzburg eine dialektale Lautvariante von täte bei der Wahl des Typs 3 intendiert wurde. ${ }^{26}$

Wenden wir uns nun der Überprüfung weiterer sozialer Faktoren zu, zunächst dem Alter der Proband*innen (Hypothese C), das sich (in Form von groben Alterskategorien) ebenfalls als statistisch signifikanter Faktor erweist $\left(\chi^{2}(12)=89,764 ; p<, 001\right.$; Cramér's V =0,082). Wie Abbildung 10 und die Kreuztabelle (Anhang B) illustriert, zeigen sich mit niedrigerem Alter eine graduelle Zunahme der analytischen Formtypen 3 und 4 (vorrangig Formtyp 3, täte-Konjunktiv) sowie eine graduelle Abnahme des Formtyps 2 (-ad/-at-Konjunktiv). Ins Auge springt insbesondere die deutliche Abnahme zwischen den Altersgruppen 20-39 und 40-59 beim täteKonjunktiv (18,0\% gegenüber 11,0\%); als affiner für analytische Formen können demnach die Generationen unter 40 verstanden werden. Dazu passt auch, dass sich umgekehrt in den älteren Generationen eine Affinität zur Synthese feststellen lässt: nicht nur die Angaben für die synthetische Dialektvariante (Formtyp 2), sondern auch für die synthetische Standardvariante (bräuchten) mehren sich, je älter die Gewährspersonen sind.

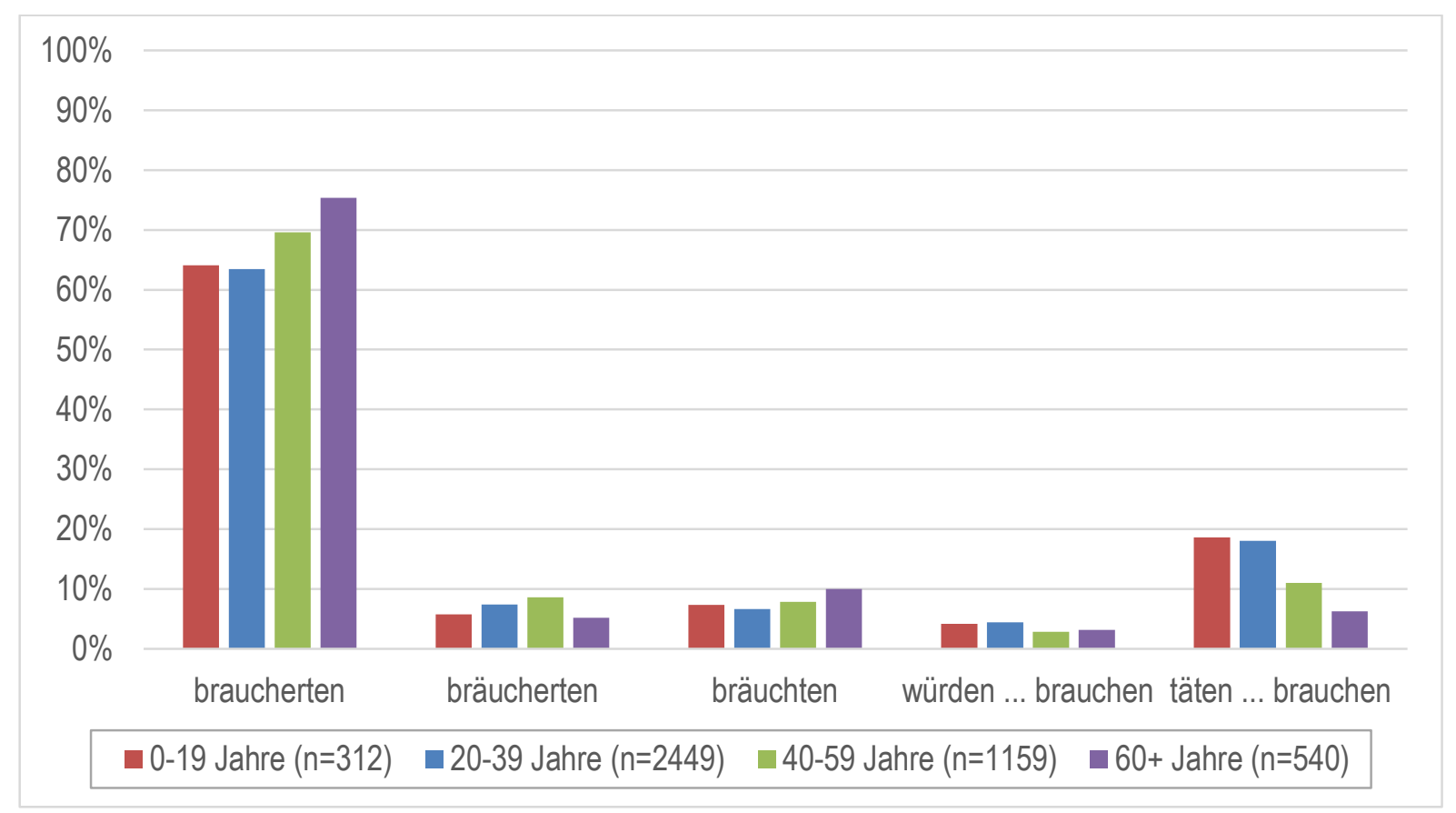

Abbildung 10: Verteilung der Konjunktiv-II-Varianten von brauchen nach Alter

Bei Hypothese E finden sich leichte, aber signifikante Effekte $\left(\chi^{2}(20)=46,060 ; p<, 01\right.$; Cramér's V =0,051; siehe Abbildung 11 und Anhang C): Tatsächlich nimmt die Angabe der Standardvarianten bräuchten und Formtyp 4 (würde-Konjunktiv) v. a. bei Studiums- und Doktoratsabschlüssen zu. Für den Formtyp 3 (täte-Konjunktiv) gilt dies nicht, was womöglich für einen erhöhten Effekt der sozialen Erwünschtheit (keine Angabe stigmatisierter Formen wie tun-Periphrasen) ${ }^{27}$ bei höherem Bildungsabschluss spricht. Zudem ist ja wahrscheinlich (siehe

\footnotetext{
${ }^{26}$ Im Unterschied zur standardnahen Realisierung in Gebieten mit stark abnehmendem Dialektgebrauch, etwa in Wien, hier besonders unter Jugendlichen, cf. Glauninger (2008: 240).

27 Zur Stigmatisierung cf. grundlegend Langer (2001).
} 
oben), dass von den Proband*innen unter den unterschiedlichen denkbaren Realisierungsformen des täte-Konjunktivs realiter nur die dialektalen Formen dat/darat/dadat vorgestellt werden - was das Stigma noch weiter begünstigt. Insgesamt erscheinen die Unterschiede zwischen den Bildungsgruppen jedoch recht gering, wie auch an der niedrigen Effektstärke ablesbar.

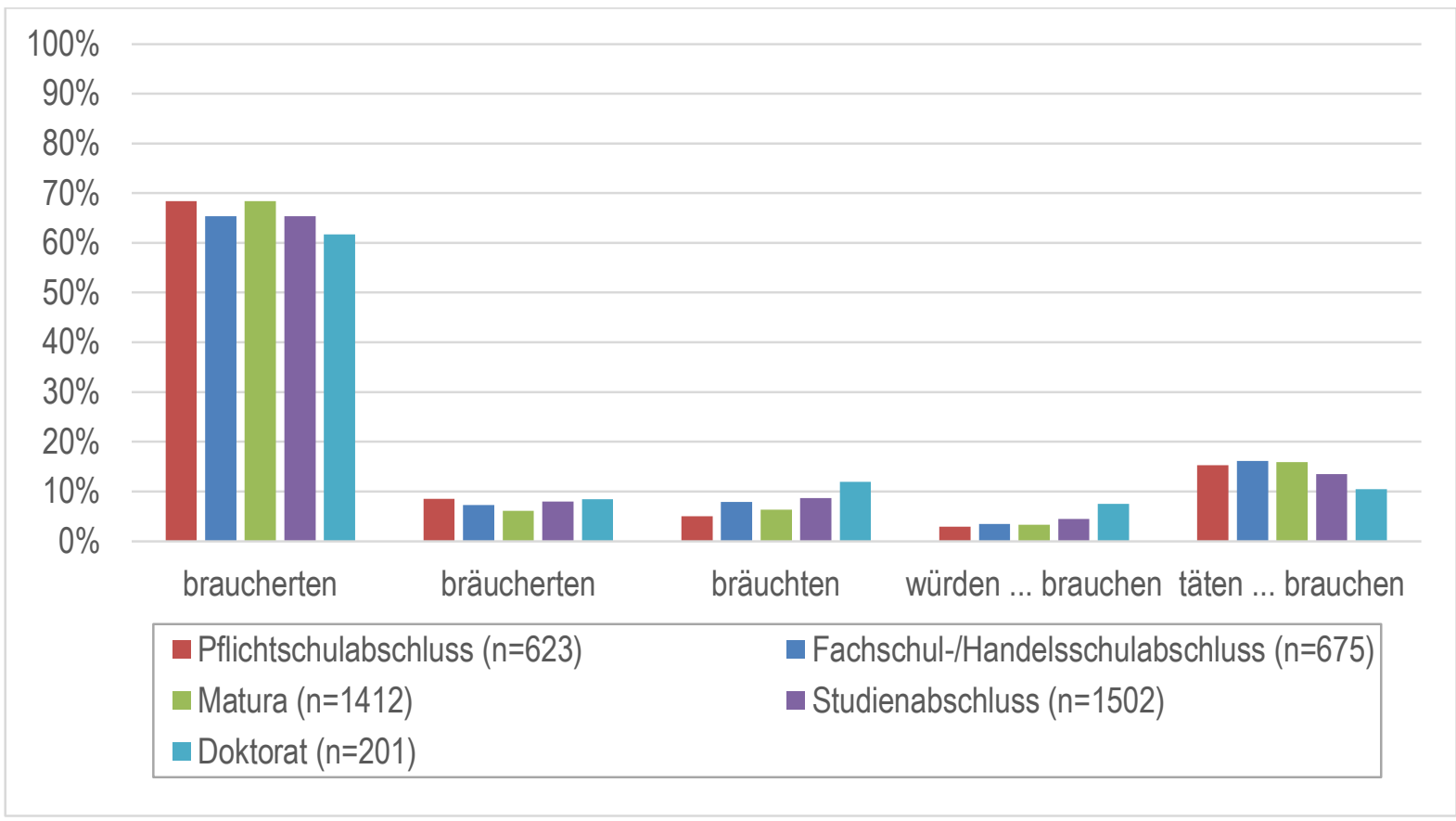

Abbildung 11: Verteilung der Konjunktiv-II-Varianten von brauchen nach Bildungsabschluss

Die Mobilität der Proband*innen haben wir durch einen dreistufigen Mobilitätsindex definiert, der zwei Kriterien (Wohndauer der Proband*innen und Sozialisierung der Eltern) berücksichtigt:

- MI1: 1.156 Proband*innen, die über 30 Jahre oder schon immer am Ort wohnen (gewohnt haben) und von denen beide Eltern im Ort oder der näheren Umgebung aufgewachsen sind

- MI2: 2.441 Proband*innen, die 10 bis 29 Jahre am Ort wohnen (gewohnt haben) und von denen mindestens ein Elternteil im Ort oder der näheren Umgebung aufgewachsen ist

- MI3: 929 Proband*innen, die weniger als 10 Jahre am Ort wohnen (gewohnt haben); zusätzlich diejenigen, die 10 bis 29 Jahre am Ort wohnen (gewohnt haben) und von denen beide Eltern nicht im Ort oder der näheren Umgebung aufgewachsen sind

Auch Hypothese F zur Mobilität bestätigt sich schließlich: Standardformen werden offenbar eher verwendet, je mobiler die Sprecher*innen sind $\left(\chi^{2}(8)=75,793 ; p<, 001\right.$; Cramér's V = 0,092; siehe Abbildung 12 und Anhang D): Bei Formtyp 4, würde-Konjunktiven, ist dieser Effekt nur gering, bei bräuchten jedoch beträchtlich (MI1 : MI2 : MI3 = 5,2\%: 6,3\%: 13,3\%). Auch wenn die einzelnen Kategorien des MI separiert werden (i. e. nur Sozialisierung der Eltern oder nur die Wohndauer der Proband*innen betrachtet werden), bestätigt sich die Hypothese. Dabei korreliert das Ergebnis auch mit dem formalen Bildungsgrad (erwartungsgemäß haben also mobilere Proband*innen tendenziell einen höheren Bildungsgrad) sowie Hauptstadtnähe (nördlichere Bezirke Salzburg-Umgebung [Flachgau] und Hallein [Tennengau] mit höherem Anteil mobilerer MI3-Proband*innen). 


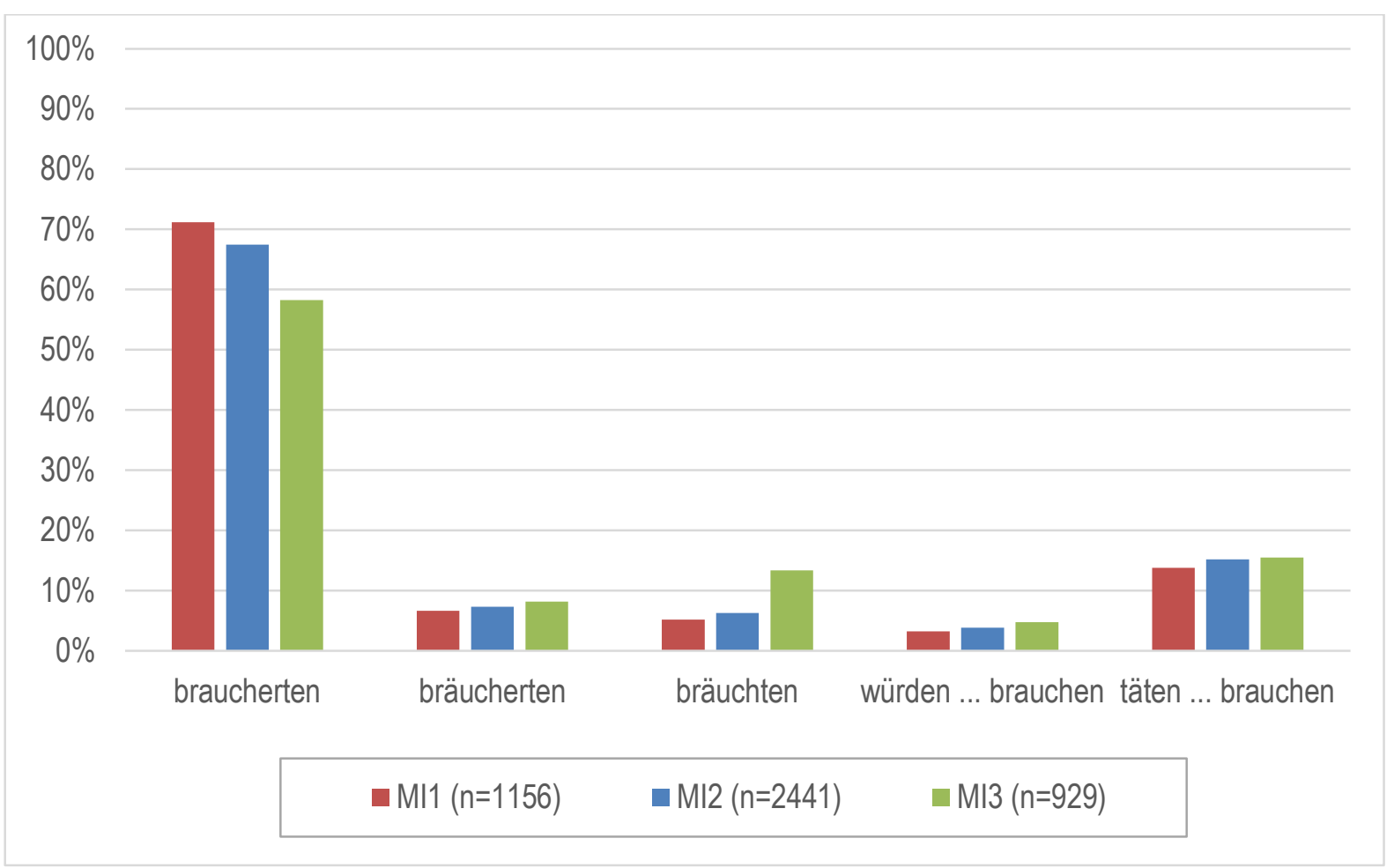

Abbildung 12: Verteilung der Konjunktiv-II-Varianten von brauchen nach Mobilitätsindex

\subsection{Zusammenfassung der Umfrageergebnisse}

Insgesamt zeichnet sich in der Analyse der möglichen Einflussvariablen in der Wahl der Varianten des Konjunktivs II beim Verb brauchen ein Zusammenspiel von mehreren Faktoren ab. Dass alle hier als mitbestimmend beschriebenen Variablen eine Rolle spielen, bestätigt sich in der multinomialen logistischen Regression (siehe Anhang E). Ausgehend von einem Regressionsmodell mit den Faktoren Geschlecht, Bildung, Bezirk, Dialektregion, Gemeindegröße und Mobilität sowie der Kovariate Alter können wir auch hier feststellen, dass sich Geschlecht, Gemeindegröße und (wenn auch nicht ganz so klar) die Dialektregion als bestimmende Faktoren ausschließen lassen. Alter, Bezirk und Mobilität tragen hingegen höchst signifikant zu einer Verbesserung des erklärten Varianzanteils bei und auch der Bildungshintergrund ist ein signifikanter Faktor.

\section{$5 \quad$ Vergleich der Ergebnisse und Ausblick}

Die hier präsentierten variationslinguistischen und dialektologischen Analysen sind freilich nur eingeschränkt miteinander vergleichbar, nicht zuletzt, weil die Daten ja nicht aufeinander abgestimmt erhoben wurden. Dennoch können wir mit aller Vorläufigkeit einzelne Aspekte an dieser Stelle kurz näher in den Blick nehmen.

Grundsätzlich lässt sich vorausschicken, dass in beiden Fallstudien eine umfassende Präferenz im Sprachgebrauch für den Formtyp (2) (-at) vorliegt - dies konnte jedoch abhängig von sozialen Faktoren im Falle der Online-Umfrage relativiert und präzisiert werden. Die dialektologisch erhobenen Daten weisen zudem darauf hin, dass bei hochfrequenten (unregelmäßigen) Verben möglicherweise eine Präferenz für synthetische Bildungsweisen herrscht. Derweil zeigt die Online-Umfrage am Beispiel brauchen, dass - wohl in Abhängigkeit von den Möglich- 
keiten der Stammmodifikation - offensichtlich auch standardnahe Formen mit dialektaler Endung (bräucherten/bräuchatn) zulässig sind.

Bezüglich weiterer Hypothesen lässt sich sagen, dass die dialektologischen Befunde die variationslinguistischen stützen (bzw. umgekehrt): Aus beiden Studien lässt sich schließen, dass analytische Formen offenbar häufiger von jüngeren Generationen verwendet werden und täte-Formen die häufigere analytische Form darstellen. So ist mittels einer Ortserhebung mit 13,2\% (414 Belege) der täte-Konjunktiv nach Formtyp 3 in seinen unterschiedlichen Realisationsvarianten die weitaus häufigste analytische Bildung. Die würde-Bildungen nach Formtyp 4 sind hier sehr selten ( $0,9 \%$ oder 27 Belege); dabei ist noch erwähnenswert, dass 25 dieser Formtyp4-Belege durch die basisdialektale Aussprache-Variante ['vu:Xt] im Lungau zustande kamen und jedenfalls nicht durch die im gesamten Erhebungsmaterial sehr seltenen und standardnäheren ['vyed $]_{\text {] }}$ bzw. ['vuet]-Periphrasen. Letzteres zeigt also den Einfluss der unterschiedlichen Methodik (gefragt waren ja dezidiert Nonstandard-Formen bzw. -Realisierungen), die sich bestätigende Tendenz unter den analytischen Konstruktionen deutet indes darauf hin, dass die Vergleichbarkeit hier möglicherweise eher gewährleistet ist als zu experimentellen Studien wie Breuer/Wittibschlager (2020: 153-154), die ein - aus Sicht unserer Ergebnisse nunmehr ungewöhnliches - Aufkommen der würde-Konjunktive feststellen. Allerdings stammen die Daten von Breuer/Wittibschlager (2020) aus dem Pongau, was die Unterschiede freilich ebenso erklären könnte. Damit erreichen wir einen weiteren Vergleichspunkt: Hypothese G aus der variationslinguistischen Studie kann auf Basis der dialektologisch erhobenen Daten ebenfalls gestützt werden: Ein Einfluss der Bezirke bzw. der kulturhistorisch relevanten Gauzugehörigkeit - im Lungau einhergehend mit Zugehörigkeit zu unterschiedlichen Dialektregionen - ist nicht von der Hand zu weisen, auch wenn die Untersuchung sich, wie eingangs erwähnt, auf zwei infrastrukturell exemplarische Regionen beschränkt hat (Tennengau, Pongau und Pinzgau blieben erhebungsbedingt unberücksichtigt). Diese kulturgeografische Strukturierung spiegelt sich auch in der Online-Umfrage, die zwar nur einen punktuellen Gebrauch (bei brauchen) behandelt, aber deren Fallzahlen noch einmal deutlich höher liegen. Besonders erwähnenswert scheinen insgesamt eine noch stärkere Tendenz zum Formtyp 2 im Lungau und Pongau und die im Wesentlichen altersunabhängige leichte Zunahme von Typ 3 (täte-Formen) im Pinzgau sowie auf Basis der dialektologischen Daten eine Präferenz für Formtyp 1 im Flachgau. Insofern müsste für das Land Salzburg künftig die Gaulandschaft in variationslinguistisch wie auch dialektologisch orientierten Ansätzen zur Konjunktivverwendung beachtet werden. Es wäre interessant, ob sich eine entsprechende Variation des Konjunktivgebrauchs auf horizontaler oder vertikaler Ebene auch in den übrigen Bundesländern mit ähnlichen Gliederungen (z. B. den Tälern in Tirol oder den historischen Vierteln in Ober- und Niederösterreich) oder in angrenzenden Regionen Südostbayerns (mit Landschaften wie dem Chiemgau, Rupertiwinkel und Berchtesgadener Land, die historisch engere Bindungen zu Salzburg aufweisen) feststellen lässt.

Für den Konjunktivgebrauch - und vertikal gedacht auch: Varietätengebrauch - im Bundesland Salzburg eröffnen unsere Ergebnisse vielversprechende Ansatzpunkte für Kontraststudien. Wie wir gehofft haben anhand zweier aktueller Forschungsprojekte zu zeigen, sind diese allemal lohnenswert. 


\section{Literaturverzeichnis}

Atlas zur Salzburger Alltagssprache (ASA). plus.ac.at/germanistik/forschung/forschungsprojekt-atlas-zur-salzburger-alltagssprache-asa/ [03.02.2022].

Bauer, Werner (1967): Lautlehre der Mundart des Lungaus mit besonderer Berücksichtigung von Zederhaus. Dissertation, Universität Wien.

Braune, Wilhelm (2004): Althochdeutsche Grammatik I. Laut- und Formenlehre. 15. Auflage, bearbeitet von Ingo Reiffenstein. Tübingen: Niemeyer. (= Sammlung kurzer Grammatiken germanischer Dialekte A, Hauptreihe, 5/1, Band 5).

Breuer, Ludwig Maximilian/Wittibschlager, Anja (2020): „The variation of the subjunctive II in Austria. Evidence from urban versus rural analyses“. Linguistic Variation 20/1 (2020): 136-171.

Britain, David (2012): „Countering the urbanist agenda in variationist sociolinguistics: dialect contact, demographic change and the rural-urban dichotomy“. In: Hansen, Sandra et al. (eds.): Dialectological and folk dialectological concepts of space. Berlin/New York, de Gruyter: 12-30. (=linguae \& litterae 17).

Donhauser, Karin (1992): Der Konjunktiv im Bairischen. In: Weiss, Andreas (eds.): Dialekte im Wandel: Referate der 4.Tagung zur bayerisch-österreichischen Dialektologie, Salzburg, 5. bis 7. Oktober 1989. Göppingen, Kümmerle: 226-242.

Hennig, Mathilde (ed.) (2016): Duden 9. Das Wörterbuch der sprachlichen Zweifelsfälle. Richtiges und gutes Deutsch. 8., vollständig überarbeitete Auflage. Berlin: Dudenverlag.

Eichhoff, Jürgen (1977-2000): Wortatlas der deutschen Umgangssprachen. 4 Bde. Bern/München: Saur.

Elspaß, Stephan/Möller, Robert (2003-): Atlas zur deutschen Alltagssprache. atlas-alltagssprache.de/ [03.02.2022].

Ender, Andrea/Kaiser, Irmtraud (2014): „Diglossie oder Dialekt-Standard-Kontinuum? Zwischen kollektiver, individueller, wahrgenommener und tatsächlicher Sprachvariation in Vorarlberg und im bairischsprachigen Österreich“. In: Huck, Dominique (ed.): Alemannische Dialektologie: Dialekte im Kontakt. Stuttgart, Steiner: 131-146. (= Zeitschrift für Dialektologie und Linguistik - Beihefte 155).

Eroms, Hans-Werner (1998): „Periphrastic tun in Present-Day Bavarian and other German Dialects“. In: Tieken-Boon van Ostade, Ingrid/van der Wal, Marijke/van Leuvensteijn, Arie (eds.): DO in English, Dutch and German. History and Present-Day Variation. Amsterdam/Münster, Stichting Neerlandistiek/Lodus Publikationem: 139-157.

Fabricius-Hansen, Cathrine (2016): „Das Verb“. In: Wöllstein, Angelika/Dudenredaktion (eds.): Duden 4. Die Grammatik. 9., vollständig überarbeitete und aktualisierte Auflage. Berlin, Dudenverlag: 395-578.

Fischer, Annette (2001): „Diachronie und Synchronie von auxiliarem tun im Deutschen“. In: Watts, Sheila/West, Jonathan/Solms, Hans-Joachim (eds.): Zur Verbmorphologie germanischer Sprachen. Tübingen, Niemeyer: 137- 154. (=Linguistische Arbeiten 446).

Fleischer, Jürg/Schallert, Oliver (2011): Historische Syntax des Deutschen. Eine Einführung. Tübingen, Narr. 
Glauninger, Manfred Michael (2008): „Synthetische und analytische „Konjunktiv 2“-Formen im Wiener Nonstandard-Deutsch“. In: Patocka, Franz/Seiler, Guido (eds.): Dialektale Morphologie, dialektale Syntax: Beiträge zum 2. Kongress der Internationalen Gesellschaft für Dialektologie des Deutschen, Wien, 20.-23. September 2006. Wien, Praesens: 233-247.

Glauninger, Manfred Michael (2011a): „Zur „,(Morpho-)Pragmatik“ von „Konjunktiv 2“-Formen im Wiener Nonstandard-Deutsch“. In: Pohl, Heinz Dieter (ed.): Akten der 10. Arbeitstagung für bayerisch-österreichische Dialektologie (Klagenfurt, September 2007). Wien, Praesens: 98-107. (= Klagenfurter Beiträge zur Sprachwissenschaft Jahrgang 34-36/20082010).

Glauninger, Manfred Michael (2011b): „Zum honorativen Konjunktiv 2 als Modularisierungsoption. Aspekte einer funktionalen Typologie des Wiener Deutsch“". In: Christen, Helen/Patocka, Franz/Ziegler, Evelyn (eds.): Struktur, Gebrauch und Wahrnehmung von Dialekt: Beiträge zum 3. Kongress der Internationalen Gesellschaft für Dialektologie des Deutschen (IGDD), Zürich, 7.-9. September 2009. Wien, Praesens: 47-57.

Höfer, Matthias (1800): Die Volkssprache in Oesterreich vorzüglich ob der Ens, nach ihrer innerlichen Verfassung und in Vergleichung mit andern Sprachen. Wien: Binz.

Kranzmayer, Eberhard (1956): Historische Lautgeographie des gesamtbairischen Dialektraumes. Graz/Köln: Böhlau.

Land Salzburg (2012): Erwerbstätige Pendler nach Bezirk. salzburg.gv.at/statistik_/Seiten/statistik_daten_wusstensiedass-erwerbspendler.aspx [03.02.2022].

Langer, Nils (2001): Linguistic Purism in Action. How auxiliary tun was stigmatized in Early New High German. Berlin/New York: de Gruyter. (= Studia linguistica Germanica 60).

Leemann, Adrian/Derungs, Curdin/Elspaß, Stephan (2019): „Analyzing linguistic variation and change using gamification web apps: The case of German-speaking Europe“. PLoS ONE 14(12): e0225399. doi.org/10.1371/journal.pone.0225399.

Lenz, Alexandra N. (2019): „Bairisch und Alemannisch in Österreich“. In: Herrgen, Joachim/Schmidt, Jürgen Erich (eds.): Sprache und Raum. Ein internationales Handbuch der Sprachvariation. Band 4: Deutsch. Unter Mitarbeit von Hanna Fischer und Brigitte Ganswindt. Berlin/Boston, de Gruyter: 318-363. (= Handbücher zur Sprach- und Kommunikationswissenschaft 30.4).

Lotze, Stefan/Gallmann, Peter (2009): „Norm und Variation beim Konjunktiv II“. In: Konopka, Marek/Strecker, Bruno (eds.): Deutsche Grammatik - Regeln, Normen, Sprachgebrauch. Berlin/New York, de Gruyter: 222-239. (=IDS-Jahrbuch 2008).

Mauser, Peter (1998): Die Morphologie im Dialekt des Salzburger Lungaus. Frankfurt a. Main/Wien: Peter Lang. (= Schriften zur deutschen Sprache in Österreich 27).

Mauser, Peter (2021a). Wiarach ba ins ret. Das Lungauer Sprachbuch. Tamsweg: Pfeifenberger.

Mauser, Peter (2021b): Wiarach ba ins ret. Die sprechende Landkarte zum Buch. https://www.pfeifenberger.at/wiarach/landkarte/ [21.09.2021].

Media-Analyse 2019/20 = Media Analyse 19/20. media-analyse.at/table/3460 [03.02.2022].

Mindl, Josef (1924/25): „Der Konjunktiv in der Mundart des oberen Landls“. Theutonista 1/2: $108-149$.

Moosbrugger, Helfried/Brandt, Holger (2020): „Itemkonstruktion und Antwortverhalten“. In: Moosbrugger, Helfried/Kevala, Augustin (eds.): Testtheorie und Fragebogenkonstruktion. 
3., vollständig neu bearbeitete, erweiterte und aktualisierte Auflage. Berlin, Springer: 6790 .

Niehaus, Konstantin (2021): „Regionale Identitäten im mehrsprachigen Bayern. Eine soziolinguistische Annäherung“. Zeitschrift für Dialektologie und Linguistik 88 (1-2/2021): 57-84.

Nowak, Jessica (2016): „Die Ablautalternanz X-O-O als partielle Regularisierungsstrategie starker Verben im Deutschen“. In: Bittner, Andreas/Köpcke, Klaus-Michael (eds.): Regularität und Irregularität in Phonologie und Morphologie. Diachron, kontrastiv, typologisch. Berlin/Boston, de Gruyter: 127-152. (= Lingua Historica Germanica 13).

Nübling, Damaris (2000): Prinzipien der Irregularisierung. Tübingen: Niemeyer. (= Linguistische Arbeiten 415).

ÖWA 2018 = Österreichische Webanalyse: ÖWA Basic, Werbeträger, Salzburger Nachrichten - Online Netzwerk. oewa.at/index.php?id=72\&offer=798\&type_id=1 [03.02.2022].

Reiffenstein, Ingo (1955): Salzburgische Dialektgeographie. Die südmittelbairischen Mundarten zwischen Inn und Enns. Giessen: Wilhelm Schmitz Verlag.

Reiffenstein, Ingo (1968): Endungszusammenfall (Suffixsynkretismus) in diachroner und synchroner Sicht. Sprache der Gegenwart V: 171-186.

Reiffenstein, Ingo (1980): Die Mundarten des Landes Salzburg. Einordnung und Überblick. Salzburger Heimatpflege - Berichte 4/3: 119-127.

Reiffenstein, Ingo (1984): Die Mundarten des Landes Salzburg. Der Lungau. Salzburger Heimatpflege-Berichte 8/1: 69-74.

Salzburg Verkehr (2021) = Salzburger Verkehrsverbund GmbH (2021): Liniennetzplan Flachgau. salzburg-verkehr.at/download/liniennetzplan-flachgau/?wpdmdl=32457\&refresh= 6023a5d8f1a3a1612948952 [03.02.2022].

Scheutz, Hannes (1999): „Umgangssprache als Ergebnis von Konvergenz- und Divergenzprozessen zwischen Dialekt und Standardsprache“. In: Stehl, Thomas (ed.): Dialektgenerationen, Dialektfunktionen, Sprachwandel. Tübingen, Narr: 105-131.

Schirmunski, Viktor M. (1962): Deutsche Mundartkunde. Vergleichende Laut- und Formenlehre der deutschen Mundarten. Aus dem Russischen übersetzt und wissenschaftlich bearbeitet von Wolfgang Fleischer. Berlin: Akademie-Verlag.

Smirnova, Elena (2006): Die Entwicklung der Konstruktion würde + Infinitiv im Deutschen. Eine funktional-semantische Analyse unter besonderer Berücksichtigung sprachhistorischer Aspekte. Berlin/New York: de Gruyter. (= Studia Linguistica Germanica 82).

Sommerauer, Verena (2009): Auszug aus der Verbalmorphologie im Dialekt des Salzburger Flachgaus - mit besonderer Berücksichtigung des Konjunktivs. Diplomarbeit, Universität Salzburg.

Steinegger, Guido (1998): Sprachgebrauch und Sprachbeurteilung in Österreich und Südtirol: Ergebnisse einer Umfrage. Frankfurt: Lang.

Vergeiner, Philip C. (2019): Kookkurrenz - Kovariation - Kontrast. Formen und Funktionen individueller Dialekt-Standardvariation in Beratungsgesprächen. Wien: Lang (= Schriften zur deutschen Sprache in Österreich 44).

Wiesinger, Peter (1989): Die Flexionsmorphologie des Verbums im Bairischen. Wien: Verlag der Österreichischen Akademie der Wissenschaften. (= Sitzungsberichte der PhilosophischHistorischen Klasse der Österreichischen Akademie der Wissenschaften 523). 
Zehetner, Ludwig (1983): „Zur mittelbairischen Flexionsmorphologie“. Zeitschrift für Dialektologie und Linguistik 50/3: 311-334.

\section{Anhang}

\begin{tabular}{|c|c|c|c|c|c|}
\hline & $\begin{array}{l}\text { SL (Salzburg- } \\
\text { Land) }\end{array}$ & HA (Hallein) & JO (St. Johann) & ZE (Zell am See) & TA (Tamsweg) \\
\hline $\begin{array}{l}\text { S } \\
\text { (Salz- } \\
\text { burg } \\
\text { Stadt) }\end{array}$ & $\begin{array}{l}\chi^{2}=122,678 \\
\mathrm{p}<, 001 \\
\text { Cramér's } V=, 208\end{array}$ & $\begin{array}{l}\chi^{2}=72,674 \\
p<, 001 \\
\text { Cramér's V=,194 }\end{array}$ & $\begin{array}{l}\chi^{2}=132,838 \\
\mathrm{p}<, 001 \\
\text { Cramér's V=,257 }\end{array}$ & $\begin{array}{l}\chi^{2}=103,238 \\
p<, 001 \\
\text { Cramér's V=,226 }\end{array}$ & $\begin{array}{l}\chi^{2}=52,290 \\
\mathrm{p}<, 001 \\
\text { Cramér's V=,175 }\end{array}$ \\
\hline SL & & $\begin{array}{l}\chi^{2}=4,623 \\
p=, 328 \\
\text { Cramér's } V=, 208\end{array}$ & $\begin{array}{l}\chi^{2}=33,772 \\
p<, 001 \\
\text { Cramér's V=,135 }\end{array}$ & $\begin{array}{l}\chi^{2}=15,612 \\
p=, 004 \\
\text { Cramér's V=,091 }\end{array}$ & $\begin{array}{l}\chi^{2}=14,856 \\
p=, 005 \\
\text { Cramér's V=,098 }\end{array}$ \\
\hline HA & & & $\begin{array}{l}\chi^{2}=14,587 \\
\mathrm{p}=, 006 \\
\text { Cramér's V=,124 }\end{array}$ & $\begin{array}{l}\chi^{2}=4,590 \\
\mathrm{p}=, 332 \\
\text { Cramér's } V=, 069\end{array}$ & $\begin{array}{l}\chi^{2}=6,922 \\
p=, 140 \\
\text { Cramér's V=,104 }\end{array}$ \\
\hline JO & & & & $\begin{array}{l}\chi^{2}=25,739 \\
\mathrm{p}<, 001 \\
\text { Cramér's V=,158 }\end{array}$ & $\begin{array}{l}\chi^{2}=7,725 \\
p=, 102 \\
\text { Cramér's V=,104 }\end{array}$ \\
\hline $\mathbf{Z E}$ & & & & & $\begin{array}{l}\chi^{2}=10,958 \\
\mathrm{p}=, 027 \\
\text { Cramér's } V=, 123\end{array}$ \\
\hline
\end{tabular}

Anhang A1: Paarvergleiche Bezirke

\begin{tabular}{|c|c|c|c|c|c|c|c|c|}
\hline & \multicolumn{6}{|c|}{ Bezirk } & \multirow[b]{2}{*}{ Gesamt } \\
\hline & & HA & $\mathrm{JO}$ & $\mathrm{S}$ & SL & TA & ZE & \\
\hline \multirow{3}{*}{$\begin{array}{l}\text { braucherten/ } \\
\text { brauchatn }\end{array}$} & Anzahl & 314 & 408 & 849 & 921 & 160 & 358 & 3010 \\
\hline & \% innerhalb von Bezirk & $71,4 \%$ & $79,8 \%$ & $56,7 \%$ & $68,2 \%$ & $80,0 \%$ & $68,1 \%$ & $66,5 \%$ \\
\hline & Standardisiertes Residuum & 1,2 & 3,7 & $-4,7$ & 8 & 2,3 &, 4 & \\
\hline \multirow{3}{*}{$\begin{array}{l}\text { bräucherten/ } \\
\text { bräuchatn }\end{array}$} & Anzahl & 31 & 14 & 133 & 98 & 13 & 42 & 331 \\
\hline & \% innerhalb von Bezirk & $7,0 \%$ & $2,7 \%$ & $8,9 \%$ & $7,3 \%$ & $6,5 \%$ & $8,0 \%$ & $7,3 \%$ \\
\hline & Standardisiertes Residuum &,- 2 & $-3,8$ & 2,2 &,- 1 &,- 4 & ,6 & \\
\hline \multirow[t]{3}{*}{ bräuchten } & Anzahl & 14 & 9 & 233 & 62 & 4 & 15 & 337 \\
\hline & $\%$ innerhalb von Bezirk & $3,2 \%$ & $1,8 \%$ & $15,6 \%$ & $4,6 \%$ & $2,0 \%$ & $2,9 \%$ & $7,4 \%$ \\
\hline & Standardisiertes Residuum & $-3,3$ & $-4,7$ & 11,5 & $-3,8$ & $-2,8$ & $-3,9$ & \\
\hline \multirow{3}{*}{$\begin{array}{l}\text { täten/dadn/ } \\
\text { dadadn ... brau- } \\
\text { chen }\end{array}$} & Anzahl & 72 & 72 & 181 & 221 & 22 & 106 & 674 \\
\hline & $\%$ innerhalb von Bezirk & $16,4 \%$ & $14,1 \%$ & $12,1 \%$ & $16,4 \%$ & $11,0 \%$ & $20,2 \%$ & $14,9 \%$ \\
\hline & Standardisiertes Residuum & \begin{tabular}{|l|l}
, 8 \\
\end{tabular} &,- 5 & $-2,8$ & 1,4 & $-1,4$ & 3,1 & \\
\hline \multirow{3}{*}{$\begin{array}{l}\text { würden ... brau- } \\
\text { chen }\end{array}$} & Anzahl & 9 & 8 & 102 & 49 & 1 & 5 & 174 \\
\hline & $\%$ innerhalb von Bezirk & $2,0 \%$ & $1,6 \%$ & $6,8 \%$ & $3,6 \%$ & $0,5 \%$ & $1,0 \%$ & $3,8 \%$ \\
\hline & Standardisiertes Residuum & $-1,9$ & $-2,6$ & 5,9 &,- 4 & $-2,4$ & $-3,4$ & \\
\hline \multirow[t]{2}{*}{ Gesamt } & Anzahl & 440 & 511 & 1498 & 1351 & 200 & 526 & 4526 \\
\hline & $\%$ innerhalb von Bezirk & $100,0 \%$ & $100,0 \%$ & $100,0 \%$ & $100,0 \%$ & $100,0 \%$ & $100,0 \%$ & $100,0 \%$ \\
\hline
\end{tabular}

Anhang A2: Kreuztabelle Bezirke (inkl. standard. Residuen) 


\begin{tabular}{|c|c|c|c|c|c|c|}
\hline & & \multicolumn{4}{|c|}{ Alterskategorien } & \multirow[b]{2}{*}{ Gesamt } \\
\hline & & $\begin{array}{l}\text { 0-19 } \\
\text { Jahre }\end{array}$ & $\begin{array}{c}20-39 \\
\text { Jahre }\end{array}$ & $\begin{array}{c}40-59 \\
\text { Jahre }\end{array}$ & $60+$ Jahre & \\
\hline \multirow{3}{*}{$\begin{array}{l}\text { braucherten/ } \\
\text { brauchatn }\end{array}$} & Anzahl & 200 & 1554 & 807 & 407 & 2968 \\
\hline & $\%$ innerhalb Alterskategorie & $64,1 \%$ & $63,5 \%$ & $69,6 \%$ & $75,4 \%$ & $66,5 \%$ \\
\hline & Standardisiertes Residuum &,- 5 & $-1,9$ & 1,3 & 2,5 & \\
\hline \multirow{3}{*}{$\begin{array}{l}\text { bräucherten/ } \\
\text { bräuchatn }\end{array}$} & Anzahl & 18 & 181 & 100 & 28 & 327 \\
\hline & $\%$ innerhalb Alterskategorie & $5,8 \%$ & $7,4 \%$ & $8,6 \%$ & $5,2 \%$ & $7,3 \%$ \\
\hline & Standardisiertes Residuum & $-1,0$ &, 1 & 1,6 & $-1,8$ & \\
\hline \multirow[t]{3}{*}{ bräuchten } & Anzahl & 23 & 163 & 91 & 54 & 331 \\
\hline & $\%$ innerhalb Alterskategorie & $7,4 \%$ & $6,7 \%$ & $7,9 \%$ & $10,0 \%$ & $7,4 \%$ \\
\hline & Standardisiertes Residuum & 0 & $-1,4$ &, 5 & 2,2 & \\
\hline \multirow{3}{*}{$\begin{array}{l}\text { täten/dadn/ } \\
\text { dadadn ... brau- } \\
\text { chen }\end{array}$} & Anzahl & 58 & 442 & 128 & 34 & 662 \\
\hline & $\%$ innerhalb Alterskategorie & $18,6 \%$ & $18,0 \%$ & $11,0 \%$ & $6,3 \%$ & $14,8 \%$ \\
\hline & Standardisiertes Residuum & 1,7 & 4,1 & $-3,4$ & $-5,2$ & \\
\hline \multirow{3}{*}{$\begin{array}{l}\text { würden ... brau- } \\
\text { chen }\end{array}$} & Anzahl & 13 & 109 & 33 & 17 & 172 \\
\hline & $\%$ innerhalb Alterskategorie & $4,2 \%$ & $4,5 \%$ & $2,8 \%$ & $3,1 \%$ & $3,9 \%$ \\
\hline & Standardisiertes Residuum & ,3 & 1,5 & $-1,7$ &,- 8 & \\
\hline \multirow[t]{2}{*}{ Gesamt } & Anzahl & 312 & 2449 & 1159 & 540 & 4460 \\
\hline & $\%$ innerhalb Alterskategorie & $100,0 \%$ & $100,0 \%$ & $100,0 \%$ & $100,0 \%$ & $100,0 \%$ \\
\hline
\end{tabular}

\section{Anhang B: Kreuztabelle Alterskategorien (inkl. standardisierte Residuen)}

\begin{tabular}{|c|c|c|c|c|c|c|c|c|}
\hline & \multicolumn{6}{|c|}{ Bildungskategorie } & \multirow[b]{2}{*}{ Gesamt } \\
\hline & & $\begin{array}{c}\text { (noch) } \\
\text { kein } \\
\text { Abschluss } \\
\end{array}$ & $\begin{array}{l}\text { Pflichtschul- } \\
\text { abschluss }\end{array}$ & $\begin{array}{l}\text { Fachschul- } \\
\text { /Handelsschul- } \\
\text { abschluss }\end{array}$ & Matura & $\begin{array}{l}\text { Studien- } \\
\text { abschluss }\end{array}$ & Doktorat & \\
\hline \multirow{3}{*}{$\begin{array}{l}\text { braucherten/ } \\
\text { brauchatn }\end{array}$} & Anzahl & 23 & 426 & 441 & 966 & 982 & 124 & 2962 \\
\hline & $\begin{array}{l}\% \text { innerhalb Bil- } \\
\text { dungskategorie }\end{array}$ & $65,7 \%$ & $68,4 \%$ & $65,3 \%$ & $68,4 \%$ & $65,4 \%$ & $61,7 \%$ & $66,6 \%$ \\
\hline & $\begin{array}{l}\text { Standardisiertes } \\
\text { Residuum }\end{array}$ &,- 1 & 5 &,- 4 & 8 &,- 6 &,- 9 & \\
\hline \multirow{3}{*}{$\begin{array}{l}\text { bräucherten/ } \\
\text { bräuchatn }\end{array}$} & Anzahl & 0 & 53 & 49 & 86 & 120 & 17 & 325 \\
\hline & $\begin{array}{l}\% \text { innerhalb Bil- } \\
\text { dungskategorie }\end{array}$ & $0,0 \%$ & $8,5 \%$ & $7,3 \%$ & $6,1 \%$ & $8,0 \%$ & $8,5 \%$ & $7,3 \%$ \\
\hline & $\begin{array}{l}\text { Standardisiertes } \\
\text { Residuum }\end{array}$ & $-1,6$ & 1,1 & 0 & $-1,7$ & 1,0 & 6 & \\
\hline \multirow[t]{3}{*}{ bräuchten } & Anzahl & 5 & 31 & 53 & 89 & 130 & 24 & 332 \\
\hline & $\begin{array}{l}\text { \% innerhalb Bil- } \\
\text { dungskategorie }\end{array}$ & $14,3 \%$ & $5,0 \%$ & $7,9 \%$ & $6,3 \%$ & $8,7 \%$ & $11,9 \%$ & $7,5 \%$ \\
\hline & $\begin{array}{l}\text { Standardisiertes } \\
\text { Residuum }\end{array}$ & 1,5 & $-2,3$ & ,4 & $-1,6$ & 1,7 & 2,3 & \\
\hline \multirow{3}{*}{$\begin{array}{l}\text { täten/dadn/ } \\
\text { dadadn ... } \\
\text { brauchen }\end{array}$} & Anzahl & 6 & 95 & 109 & 224 & 203 & 21 & 658 \\
\hline & $\begin{array}{l}\text { \% innerhalb Bil- } \\
\text { dungskategorie }\end{array}$ & $17,1 \%$ & $15,2 \%$ & $16,1 \%$ & $15,9 \%$ & $13,5 \%$ & $10,4 \%$ & $14,8 \%$ \\
\hline & $\begin{array}{l}\text { Standardisiertes } \\
\text { Residuum }\end{array}$ & ,4 & ,3 & ,9 & 1,0 & $-1,3$ & $-1,6$ & \\
\hline \multirow{3}{*}{$\begin{array}{l}\text { würden ... } \\
\text { brauchen }\end{array}$} & Anzahl & 1 & 18 & 23 & 47 & 67 & 15 & 171 \\
\hline & $\begin{array}{l}\% \text { innerhalb Bil- } \\
\text { dungskategorie }\end{array}$ & $2,9 \%$ & $2,9 \%$ & $3,4 \%$ & $3,3 \%$ & $4,5 \%$ & $7,5 \%$ & $3,8 \%$ \\
\hline & $\begin{array}{l}\text { Standardisiertes } \\
\text { Residuum }\end{array}$ &,- 3 & $-1,2$ &,- 6 & $-1,0$ & 1,2 & 2,6 & \\
\hline \multirow[t]{2}{*}{ Gesamt } & Anzahl & 35 & 623 & 675 & 1412 & 1502 & 201 & 4448 \\
\hline & $\begin{array}{l}\text { \% innerhalb Bil- } \\
\text { dungskategorie }\end{array}$ & $100,0 \%$ & $100,0 \%$ & $100,0 \%$ & $100,0 \%$ & $100,0 \%$ & $100,0 \%$ & $100,0 \%$ \\
\hline
\end{tabular}

Anhang C: Kreuztabelle Bildungskategorien (inkl. standardisierte Residuen) 


\begin{tabular}{|c|c|c|c|c|c|}
\hline & \multicolumn{3}{|c|}{ Mobilitätsindex } & \multirow[b]{2}{*}{ Gesamt } \\
\hline & & 1 & 2 & 3 & \\
\hline \multirow{3}{*}{ braucherten/brauchatn } & Anzahl & 823 & 1646 & 541 & 3010 \\
\hline & \% innerhalb von Mobilitätsindex & $71,2 \%$ & $67,4 \%$ & $58,2 \%$ & $66,5 \%$ \\
\hline & Standardisiertes Residuum & 2,0 & 6 & $-3,1$ & \\
\hline \multirow[t]{3}{*}{ bräucherten/bräuchatn } & Anzahl & 77 & 178 & 76 & 331 \\
\hline & \% innerhalb von Mobilitätsindex & $6,7 \%$ & $7,3 \%$ & $8,2 \%$ & $7,3 \%$ \\
\hline & Standardisiertes Residuum &,- 8 & 0 & 1,0 & \\
\hline \multirow[t]{3}{*}{ bräuchten } & Anzahl & 60 & 153 & 124 & 337 \\
\hline & $\%$ innerhalb von Mobilitätsindex & $5,2 \%$ & $6,3 \%$ & $13,3 \%$ & $7,4 \%$ \\
\hline & Standardisiertes Residuum & $-2,8$ & $-2,1$ & 6,6 & \\
\hline \multirow{3}{*}{$\begin{array}{l}\text { täten/dadn/dadadn ... } \\
\text { brauchen }\end{array}$} & Anzahl & 159 & 371 & 144 & 674 \\
\hline & \% innerhalb von Mobilitätsindex & $13,8 \%$ & $15,2 \%$ & $15,5 \%$ & $14,9 \%$ \\
\hline & Standardisiertes Residuum & $-1,0$ & 4 & ,5 & \\
\hline \multirow[t]{3}{*}{ würden ... brauchen } & Anzahl & 37 & 93 & 44 & 174 \\
\hline & \% innerhalb von Mobilitätsindex & $3,2 \%$ & $3,8 \%$ & $4,7 \%$ & $3,8 \%$ \\
\hline & Standardisiertes Residuum & $-1,1$ &,- 1 & 1,4 & \\
\hline \multirow[t]{2}{*}{ Gesamt } & Anzahl & 1156 & 2441 & 929 & 4526 \\
\hline & \% innerhalb von Mobilitätsindex & $100,0 \%$ & $100,0 \%$ & $100,0 \%$ & $100,0 \%$ \\
\hline
\end{tabular}

Anhang D: Kreuztabelle Mobilitätsindex

\begin{tabular}{|l|l|l|l|}
\hline Prädiktor & $\chi^{2}$ & $\mathrm{df}$ & $\mathrm{p}$ \\
\hline Konstanter Term &, 000 & 0 & $\cdot$ \\
\hline Alter & 87,717 & 5 &, 000 \\
\hline Bezirk & 144,050 & 25 &, 000 \\
\hline Geschlecht & 3,476 & 5 &, 627 \\
\hline Dialektregion & 18,851 & 15 &, 221 \\
\hline Mobilitätsindex & 35,244 & 10 &, 000 \\
\hline höchster Bildungsabschluss & 45,785 & 30 &, 033 \\
\hline Gemeindegröße & 14,618 & 10 &, 147 \\
\hline
\end{tabular}

Modellanpassung: Vergleich zum Modell mit nur dem konstanten Term: $\chi^{2}(100)=543,744 ; \mathrm{p}<, 001$; Nagelkerke $\mathrm{R}^{2}=, 129$

Anhang E: Regressionsmodell 